\title{
ARTICLE
}

\section{A dual positional specific lipoxygenase functions in the generation of flavor compounds during climacteric ripening of apple}

\author{
Doreen Schiller ${ }^{1}$, Carolina Contreras ${ }^{2}$, Jörg Vogt ${ }^{3}$, Frank Dunemann ${ }^{4}$, Bruno G Defilippi ${ }^{5}$, Randolph Beaudry ${ }^{2}$ and Wilfried Schwab $^{1}$
}

Lipoxygenase (LOX) is an important contributor to the formation of aroma-active C 6 aldehydes in apple (Malus $\times$ domestica) fruit upon tissue disruption but little is known about its role in autonomously produced aroma volatiles from intact tissue. We explored the expression of 22 putative LOX genes in apple throughout ripening, but only six LOXs were expressed in a ripening-dependent manner. Recombinant LOX1:Md:1a, LOX1:Md:1c, LOX2:Md:2a and LOX2:Md:2b proteins showed 13/9-LOX, 9-LOX, 13/9-LOX and 13-LOX activity with linoleic acid, respectively. While products of LOX1:Md:1c and LOX2:Md:2b were S-configured, LOX1:Md:1a and LOX2:Md:2a formed 13(R)-hydroperoxides as major products. Site-directed mutagenesis of Gly567 to an alanine converted the dual positional specific LOX1:Md:1a to an enzyme with a high specificity for 9(S)-hydroperoxide formation. The high expression level of the corresponding MdLOX1a gene in stored apple fruit, the genetic association with a quantitative trait locus for fruit ester and the remarkable agreement in regio- and stereoselectivity of the LOX1:Md:1a reaction with the overall LOX activity found in mature apple fruits, suggest a major physiological function of LOX1:Md:1a during climacteric ripening of apples. While LOX1:Md:1c, LOX2:Md:2a and LOX2:Md:2b may contribute to aldehyde production in immature fruit upon cell disruption our results furnish additional evidence that LOX1:Md:1a probably regulates the availability of precursors for ester production in intact fruit tissue.

Horticulture Research (2015) 2, 15003; doi:10.1038/hortres.2015.3; Published online: 25 February 2015

\section{INTRODUCTION}

Flavor is one of the most important quality attributes of the ripe apple fruit and plays a decisive role in the economic prospects of a cultivar in the market. Among the 350 volatile compounds that are emitted by apple fruit, ${ }^{1}$ about 20 are considered as key character compounds as they dominate the typical aroma of a particular cultivar. ${ }^{2}$ Substances with a high impact on the aroma of apple are esters, such as butyl acetate, hexyl acetate and 2-methyl-butyl acetate, as well as various C6 alcohols and aldehydes. Although aldehydes are produced at relatively low amounts in mature apples, they determine the flavor of immature fruits. ${ }^{3}$ During ripening, the aroma profile changes from aldehydes to esters, ${ }^{4}$ to such an extent that esters can account for more than $80 \%$ of all aroma-relevant substances in some cultivars. ${ }^{5}$

The formation of primary metabolites for inclusion into fruit esters occurs via several pathways. ${ }^{6}$ Fatty acid degradation is thought to contribute to the formation of short chain fatty acids and aldehydes to yield straight-chain esters. ${ }^{7-9}$ The leucine/isoleucine/valine pathway provides precursors to branched-chain esters ${ }^{9}$ and may contribute to the formation of short-chain fatty acids. ${ }^{10}$ Saturated fatty acids are degraded by the enzymatic steps of $\beta$ oxidation whereas linoleic (LA) and linolenic acid ( $L n A)$ are either catabolized through $\beta$-oxidation or the lipoxygenase (LOX) pathway. The latter involves the sequential action of LOX, hydroperoxide lyase and alcohol dehydrogenase (ADH). ${ }^{6}$ The resulting alcohols are linked to an acyl moiety by alcohol acyl-CoA transferases (AATs) to form esters. Unsaturated straight-chain esters appear to arise mainly by the LOX pathway in apple.

LOX (linoleate oxygen oxidoreductase, EC 1.13.11.12) are nonheme iron-containing dioxygenases. They catalyse the regio- and stereospecific introduction of molecular dioxygen into polyunsaturated fatty acids, containing a Z,Z-1,4-pentadiene system such as LA and LnA to form hydroperoxy octadecadienoic acid (HpODE) and hydroperoxy octadecatrienoic acid, respectively. ${ }^{11}$ Plant LOX are classified according to the position of oxygenation in the carbon backbone of LA. ${ }^{12}$ This occurs either at carbon atom 9 (9-LOX) or 13 (13-LOX), forming the corresponding hydroperoxy derivatives, respectively (Figure 1). However, LOX enzymes are not perfectly specific and biocatalysts that produce more than $10 \%$ of the alternative regio-isomer are called dual positional specific LOX. ${ }^{14}$ Both 9- and 13-hydroperoxides can be catabolized to aroma-active volatile aldehydes by hydroperoxide lyase. However, only 13-LOX activity contributes to the apple aroma due to the formation of precursors of $\mathrm{C} 6$ volatile compounds. ${ }^{15}$ Alternatively, LOX genes are classified based on comparison of LOX primary structures. ${ }^{13}$ Most plant LOX share high sequence similarity of about $70 \%$ and are therefore grouped into the so-called type-1 LOX class. Members of the type-2 LOX class show only moderate sequence similarity but exhibit a putative chloroplast transit peptide. ${ }^{12}$ TomloxC, a chloroplast-targeted LOX from tomato, has been identified as key LOX specifically involved in the generation of fatty acid-derived C6 aldehydes and alcohols upon tissue disruption. ${ }^{16}$

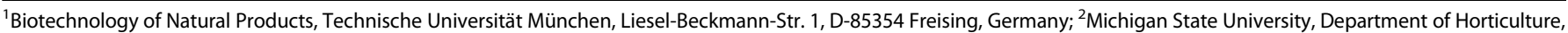

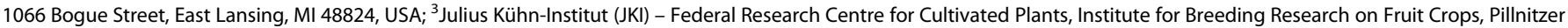

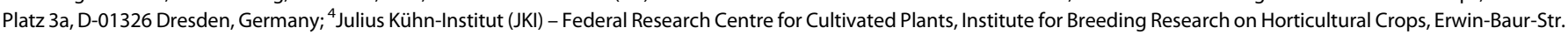
27, D-06484 Quedlinburg, Germany and ${ }^{5}$ INIA La Platina, PO Box 439-9, Santiago, Chile

Correspondence: W Schwab (wilfried.schwab@tum.de)

Received: 13 November 2014; Revised: 9 January 2015; Accepted: 21 January 2015 


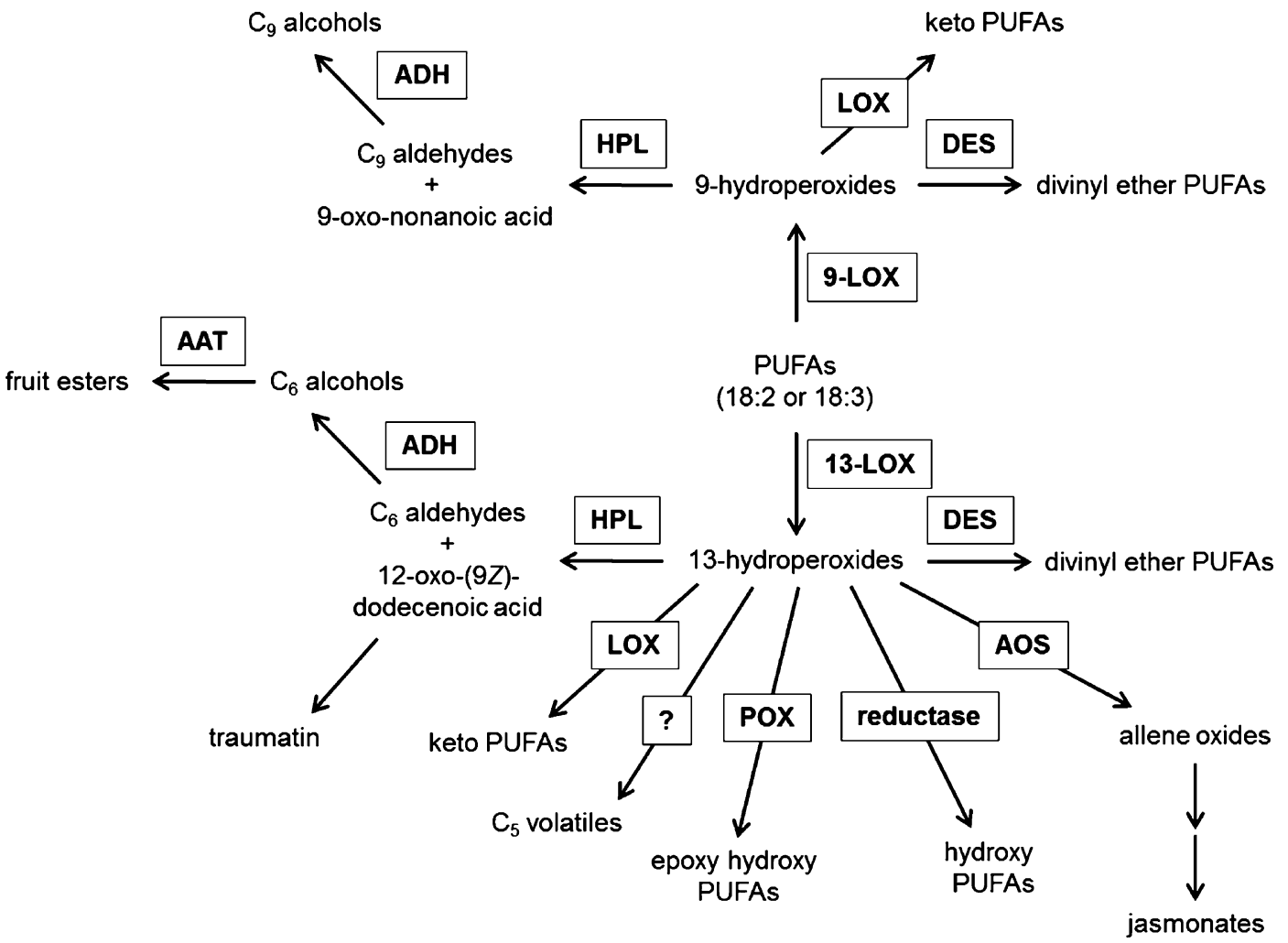

Figure 1. The LOX pathway. The dioxygenation of PUFAs by 9- and 13-LOX activity forms precursors for important phytooxylipins with functions in plant defense, wound signaling, senescence and fruit ripening. The formation of fruit esters from 13-hydroperoxide occurs by the HPL pathway and the action of AAT. AAT, alcohol acyl-CoA transferase; ADH, alcohol dehydrogenase; AOS, allene oxid synthase; DES, divinyl ether synthase; $\mathrm{HPL}$, hydroperoxide lyase; POX, peroxygenase, PUFA, polyunsaturated fatty acid. Adapted from Feussner and Wasternack. ${ }^{13}$

Plant LOXs form multi-gene families comprising varying numbers of genes. While five LOX genes have been cloned from the transcriptome of tomato, ${ }^{16}$ up to 20 LOXs have been reported for soybean ${ }^{17}$ and grapevine. ${ }^{18}$ It has been postulated that plant LOX may be involved in plant growth, development, senescence, stress, defence and response to wounding. ${ }^{13}$ LOX enzymes catalyze the initial reaction in the biosynthetic pathways of diverse defense molecules such as jasmonates and traumatin, and of volatile compounds such as hexanal, hexenal and hexenol (Figure 1). LOX genes with potential roles during fruit ripening have been described in tomato, ${ }^{19}$ apricot, $^{20}$ peach $^{21,22}$ and kiwifruit. ${ }^{23}$

Initially, the number of putative LOX genes in apple was determined to be nine ${ }^{9}$ but was recently revised to 23 by detailed in silico analysis of the Malus $\times$ domestica cv. Golden Delicious genome sequence. ${ }^{24}$ The identification of genes putatively involved in apple fruit development and flavor formation revealed a LOX gene (expressed sequence tag (EST) cluster MD187410, MdLOX1a ${ }^{24}$ ) through large-scale statistical analysis of apple EST libraries. ${ }^{25}$ This gene was later mapped on the apple linkage group 9 closely to a quantitative trait locus cluster for ester-type volatiles. ${ }^{26} \mathrm{MdLOX} 1 \mathrm{a}$ transcript level increases during climacteric ripening and storage of apple fruit independent of the tested apple cultivar. ${ }^{24,25}$ The gene is also expressed at high levels in young leaves and is upregulated in response to ethylene consistent with its putative function in the formation of hexanal-derived esters (i.e., hexyl esters) during fruit ripening. ${ }^{9}$

Despite the extensive research on ripening-dependent changes in the aroma profile of apple fruits, very little information is available on the function and regulation of enzymes involved. To identify LOX enzymes contributing to 6-carbon straight-chain ester formation, we biochemically analyzed four LOX enzymes of the MdLOX1 and
MdLOX2 gene cluster, as their genes show a ripening-related expression pattern. ${ }^{24}$ Here we report the characterization of a dual positional specific $(R)$-LOX, which is induced by ethylene in fruits and forms both 13(R)- and 9(S)-hydroperoxides from LA and LnA. Sitedirected mutagenesis elucidated the sources of enzymatic specificity. The relevance of the candidate gene for fruit ester production in apple fruit is discussed in the context of available information on ripening-dependent changes in aroma composition.

\section{MATERIALS AND METHODS}

\section{Chemicals}

All chemicals used were standard commercial products of analytical grade from the following companies: linoleic acid (Roth, Karlsruhe, Germany); $\alpha$ linolenic acid (Roth); arachidonic acid (Sigma-Aldrich, Steinheim, Germany); triphenyl phosphine (Merck Schuchardt, Hohenbrunn, Germany); HPLC solvents (Mallinckrodt Baker, Deventer, Holland); $9( \pm)$-HODE und 13( \pm )-HODE (Cayman Chemical, Tallinn, Estonia); Hydroperoxy HPLC Mix (Cayman Chemical).

\section{Cloning of full-length apple LOX cDNAs}

Total RNA was isolated from fruit peel of domesticated apple (Malus $X$ domestica Borkh.) cultivar Golden Delicious by CTAB extraction. ${ }^{27}$ Reverse transcription was performed on $5 \mu \mathrm{g}$ of total RNA with Superscript III reverse transcriptase (Invitrogen, Karlsruhe, Germany) and a GeneRacer oligo-dT primer (5'-GCT GTC AAC GAT ACG CTA CGT AAC GGC ATG ACA GTG T(18)$\left.3^{\prime}\right)$. The primers for gene amplification were designed based on bioinformatically selected LOX sequences from the apple genome described by Vogt et al. ${ }^{24}$ In total, primer pairs for six full-length apple LOX genes with confirmed presence of all functional motifs were designed (Supplementary Table S1). Amplification of LOX genes was achieved via Phusion ${ }^{\circledR}$ highfidelity DNA polymerase (Thermo Fisher Scientific, St Leon-Rot, Germany). The temperature program used was $60 \mathrm{~s}$ at $98{ }^{\circ} \mathrm{C}, 1$ cycle; $10 \mathrm{~s}$ at $95{ }^{\circ} \mathrm{C}, 30 \mathrm{~s}$ 
at corresponding annealing temperature (Supplementary Table S1), $90 \mathrm{~s}$ at $72{ }^{\circ} \mathrm{C}, 35$ cycles; final extension at $72{ }^{\circ} \mathrm{C}$ for $5 \mathrm{~min}$. PCR products were then gel-purified and A-tailed with Taq-DNA polymerase (New England Biolabs, Frankfurt, Germany) for ligation into the PGEM-T easy vector (Promega, Mannheim, Germany). Sequencing was used to confirm the identity of the amplified fragments.

\section{Characterization of ripening apple fruit}

'Jonagold' (Malus $\times$ domestica Borkh.) apples from a commercial orchard in Sparta, MI were used to investigate the relationship between stage of development, the volatile profile and the expression of putative LOX genes as described ${ }^{7}$ (Supplemental Figure S10).

\section{RNA isolation and reverse transcription PCR (RT-PCR)}

The expression of putative $L O X$ genes was initially evaluated using RT-PCR to screen candidate genes for further analysis. A hot borate extraction procedure was used ${ }^{28}$ with some adaptation using a commercial purification kit (RNeasy Mini Kit Qiagen). Two micrograms of total RNA was pretreated with RNAse-free DNase (Qiagen Inc., Valencia, CA, USA) to remove contaminating genomic DNA. The concentration of total RNA was measured using a spectrophotometer (Nanodrop; Thermo Scientific). First-strand cDNA was synthesized from $2.0 \mu \mathrm{g}$ of treated total RNA, using a commercial kit as described by the manufacturer (SuperScript III; Invitrogen, Carlsbad, CA, USA) to a total volume of $20 \mu \mathrm{L}$. Expression of the LOXs was determined relative to the housekeeping gene glyceraldehyde 3-phosphate dehydrogenase (GAPDH) using semi-quantitative PCR analysis. CDNA $(1 \mu \mathrm{L})$ were used as templates in a $25-\mu \mathrm{L}$ reaction containing $10 \mu \mathrm{M}$ of the forward and reverse gene-specific primer (Supplementary Table S2). Specific and not conserved regions from each of 22 putative $L O X$ genes were amplified. Amplicon sizes ranged from 196 to $409 \mathrm{bp}$. For each PCR, two biological and two technical replicates were analyzed. PCR primers were designed using Primer3. ${ }^{29}$ Primer sequences, the expected size of the PCR product, optimum cycle number and optimum temperature for primer binding are listed (Supplementary Table S2). The PCR reaction was performed as follows: (i) 2 min at $94{ }^{\circ} \mathrm{C}$, (ii) $30 \mathrm{~s}$ at $94{ }^{\circ} \mathrm{C}$, (iii) $30 \mathrm{~s}$ at 55-61 ${ }^{\circ} \mathrm{C}$, and (iv) $45 \mathrm{~s}$ at $72{ }^{\circ} \mathrm{C}$, repeating 19-33 cycles from steps 2 to 4 , and final elongation 5 min at $72{ }^{\circ} \mathrm{C}$. Two genes, MdLOX7d and MdLOX 16 , were amplified using Touchdown-PCR with the following steps: (i) 5 min at $94{ }^{\circ} \mathrm{C}$, (ii) $30 \mathrm{~s}$ at $94{ }^{\circ} \mathrm{C}$, (iii) $30 \mathrm{~s}$ at $72{ }^{\circ} \mathrm{C}$ (lowering $1{ }^{\circ} \mathrm{C}$ every cycle), (iv) $45 \mathrm{~s}$ at $72{ }^{\circ} \mathrm{C}$, (v) repeating nine cycles from steps 2 to 4 , (vi) $30 \mathrm{~s}$ at $94{ }^{\circ} \mathrm{C}$, (vii) $30 \mathrm{~s}$ at $62{ }^{\circ} \mathrm{C}$, (viii) $45 \mathrm{~s}$ at $72{ }^{\circ} \mathrm{C}$, and (ix) repeating 31-33 cycles from steps 6 to 8 , and final 5 -min elongation at $72{ }^{\circ} \mathrm{C}$. The amplified PCR products were separated by electrophoresis on a $1.2 \%(\mathrm{w} /$ v) agarose gel, visualized with UV fluorescence of ethidium bromide, and photographed. Relative light density of the bands was quantified by a digital imaging system (EagleEye II; Stratagene, La Jolla, CA, USA). To identify the optimum cycle, the gene products amplified by PCR had to be visible on the gel electrophoresis and be quantifiable by light density measurement without saturation of pixels. The number of PCR cycles needed ranged from 20 to 34 (Supplementary Table S2). PCR products were cleaned using ExoSAP-IT ${ }^{\circledR}$ for PCR product cleanup (Affymetrix, Santa Clara, CA, USA) and sequenced at RTSF (MSU Genomics Core, East Lansing, MI, USA) to verify identity. Expression data for all LOX genes were normalized across developmental stages and relative to the control gene GAPDH. To do this, the maximal band density for all eight stages was taken as 1 and the remaining seven readings expressed as a fraction of the maximum. The data for GAPDH was handled similarly. The ratio was then taken between the $L O X$ fraction and the GAPDH fraction to normalize relative to GAPDH abundance. The spot density for the $G A P D H$ varied approximately $\pm 13 \%$ across the eight developmental stages.

Real-time quantitative PCR (qPCR) assays

Six of the putative LOX genes (MdLOX4a, MdLOX6a and MDLOX6b in the 13LOX group and MdLOX1a, MdLOX7a and MdLOX7C in the 9-LOX group) were selected for qPCR analysis based on RT-PCR results indicating significant changes in expression during ripening. In addition, an additional 13-LOX candidate gene (MdLOX2a) was subjected to qPCR based on its apparent ripening-independent RT-PCR expression pattern in order to further validate RT-PCR results. For the selected LOX genes, as well as for the housekeeping gene, GAPDH, PCR primers (Supplementary Table S3) were designed using Primer Express Software for Real Time PCR version 3.0 (Applied Biosystems, Grand Island, NY, USA) according to the strategies set up by Yokoyama and Nishitani. ${ }^{30}$ The high level of sequence similarity between the apple LOX members was taken into account when designing quantitative real-time PCR ( $\mathrm{PPCR}$ ) primers to avoid cross-annealing of primers. For this, the gene-specificity of primer sets was tested using the following procedure: (i) Genome Database for Rosaceae NCBI BLAST searches of apple genome database were performed for each primer to confirm that no other sequence in the apple genome was similar to any primer; and (ii) the specificity of PCR amplification was examined by monitoring the dissociation curves for $\mathrm{QPCR}$ reactions using the ABI PRISM 7900HT sequence detection system (Applied Biosystems). PCR amplification efficiencies for each primer were plotted in a semi-log regression plot, all primers efficient primers were in a range of $-3.32 \pm 10 \%$ slope $(-3.32$ indicates a PCR reaction with $100 \%$ efficiency). The length of all PCR products ranged from 50 to $70 \mathrm{bp}$. Final primer sets are listed in Supplementary Table S3. Serial dilutions of CDNA (10, 1, 0.1 and

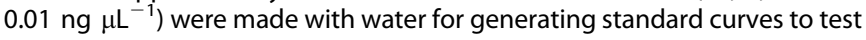
primer efficiency. PCR reactions were performed in a total volume of $10 \mu \mathrm{L}$, using $3 \mu \mathrm{L}$ of the two primers $(1 \mu \mathrm{M}), 5 \mu \mathrm{L}$ of $2 \times$ SYBR Green PCR Master Mix (Applied Biosystems) and $2 \mu \mathrm{L}$ of the cDNA template. qPCR analysis was on an ABI PRISM 7900HT sequence detection system (Applied Biosystems). The qPCR program included a preliminary step of $2 \mathrm{~min}$ at $50{ }^{\circ} \mathrm{C}$ and $10 \mathrm{~min}$ at $95{ }^{\circ} \mathrm{C}$, followed by 40 cycles of $95{ }^{\circ} \mathrm{C}$ for $15 \mathrm{~s}$ and $60{ }^{\circ} \mathrm{C}$ for $1 \mathrm{~min}$. No-template controls for each primer pair were included in each run. Apple GAPDH gene was used as an internal control to normalize small differences in template amounts. The real time data generated was analyzed applying the $\Delta \Delta C_{t}$ method. $^{31}$ At least two different RNA isolations and CDNA syntheses were used as biological replicates and three technical replicates for the qPCR. Expression levels produced by qPCR were expressed as a ratio relative to the fruit stage one to emphasize ripening-dependent behavior.

\section{Expression of recombinant LOX protein in yeast}

Heterologous expression of four LOX genes in Saccharomyces cerevisiae was achieved by cloning in the pYES2 expression vector (Invitrogen, Karlsruhe, Germany). The full-length open reading frames of the genes were amplified from the pGEM-T easy vector constructs described above with Phusion ${ }^{\circledR}$ High-Fidelity DNA Polymerase. Since the pYES2 vector does not exhibit a tag for protein purification, the PCR products were subcloned in the bacterial pRSET B vector (Invitrogen, Karlsruhe, Germany). This vector enables in frame fusion to a sequence that encodes an $\mathrm{N}$-terminal fusion peptide including a polyhistidine tag and an enterokinase cleavage recognition site for subsequent removal of the fusion peptide from the purified recombinant protein. The sequences of the fusion proteins were then amplified from the PRSET B vector with oligonucleotides containing the yeast translation initiation site required for heterologous expression in yeast and cloned in the pYES2 expression vector. Oligonucleotides applied during this procedure are depicted in Supplementary Table S5. The resulting vector constructs were transformed into the $S$. cerevisiae INVSC1 strain for expression of recombinant protein, which was performed as described by. ${ }^{32}$ Cells were harvested $24 \mathrm{~h}$ after induction in aliquots corresponding to $25 \mathrm{~mL}$ culture by centrifugation $\left(5000 \mathrm{~g}, 4{ }^{\circ} \mathrm{C}, 5 \mathrm{~min}\right)$. Cell pellets were washed with water once, frozen in liquid nitrogen and kept at $-80{ }^{\circ} \mathrm{C}$ until further use.

\section{Partial purification of recombinant protein}

Cell pellets corresponding to $200 \mathrm{~mL}$ culture were resuspended in $4 \mathrm{~mL}$ of breaking buffer (50 mM sodium phosphate buffer, pH 7.5, 1 mM EDTA, 5\% (v/v) glycerol, $10 \mathrm{mM}$ imidazole, $1 \mathrm{mM}$ PMSF) to obtain an $\mathrm{OD}_{600}$ of 50 . Lysis of cells was achieved by addition of glass beads and repeated vortexing for $30 \mathrm{~s}$ followed by $30 \mathrm{~s}$ incubation on ice. After $20 \mathrm{~min}$ of treatment cell debris was removed by centrifugation $\left(14.800 \mathrm{~g}, 4{ }^{\circ} \mathrm{C}, 10 \mathrm{~min}\right)$. Protein crude extract was loaded on a gravity column with $1 \mathrm{~mL}$ Profinity ${ }^{\mathrm{TM}}$ IMAC Ni-charged Resin (Bio-Rad Laboratories, München, Germany) and incubated for $2 \mathrm{~h}$ with orbital shaking at $4{ }^{\circ} \mathrm{C}$. After washing with $10 \mathrm{~mL}$ of $50 \mathrm{mM}$ sodium phosphate buffer (pH 7.5) containing $300 \mathrm{mM} \mathrm{NaCl}$ and $30 \mathrm{mM}$ imidazole, elution was performed with $600 \mu \mathrm{L}$ of $50 \mathrm{mM}$ sodium phosphate buffer (pH 7.5) containing $300 \mathrm{mM} \mathrm{NaCl}$ and $500 \mathrm{mM}$ imidazole. The elution was repeated three more times. Fractions with LOX activity were pooled, dialyzed overnight against $50 \mathrm{mM}$ sodium phosphate buffer $(\mathrm{pH} 7.0)$ and concentrated by ultrafiltration using Vivaspin 500 centrifugal concentrators (Sartorius Stedim Biotech, Göttingen, Germany) with a molecular weight cutoff of 50000 . Samples were analyzed via SDS-PAGE and protein concentration was determined by the Bradford method using bovine serum albumin as standard. ${ }^{33}$ Protein was stored in small aliquots at $-80{ }^{\circ} \mathrm{C}$ until required.

Determination of enzyme properties

LOX activity was determined by continuously monitoring the formation of conjugated dienes at $234 \mathrm{~nm}$. The standard assay mixture consisted of 
$500 \mu \mathrm{L} 50 \mathrm{mM}$ sodium phosphate buffer ( $\mathrm{pH}$ 7.5) and the appropriate amount of purified protein $(0.2$ to $2 \mu \mathrm{g})$. Reactions were started by addition of $0.3 \mathrm{mM}$ substrate and incubated at $25^{\circ} \mathrm{C}$ with constant shaking for $1 \mathrm{~h}$. The reaction products were extracted with dichloromethane, reduced by addition of $400 \mathrm{nmol}$ triphenyl phosphine and evaporated to dryness under a constant flow of nitrogen. After resuspension in 30\% (v/v) methanol samples were submitted to HPLC analysis. Reaction buffers were prepared with $0.5 \mathrm{pH}$ unit increments as follows: $50 \mathrm{mM}$ sodium citrate buffer $(\mathrm{pH} 3.0-6.0)$, $50 \mathrm{mM}$ sodium phosphate buffer ( $\mathrm{pH}$ 6.0-8.0) and $50 \mathrm{mM}$ Tris $\mathrm{HCl}$ buffer ( $\mathrm{pH}$ 8.0-10.0). For determination of the $\mathrm{pH}$ optimum of the recombinant proteins, the reaction was run across a pH range of $3.0-10.0$ at $25{ }^{\circ} \mathrm{C}$. The optimum temperature was determined by assaying LOX activity rates at temperatures of $5-55{ }^{\circ} \mathrm{C}$ with $5{ }^{\circ} \mathrm{C}$ increments at a pH of 7.0. Kinetic properties of recombinant LOX were determined by monitoring hydroperoxide formation across a range of concentrations of LA and LnA with a Nicolet evolution 100 spectrophotometer (Thermo, Cambridge, UK). The standard assay was performed at $45{ }^{\circ} \mathrm{C}$ with $0-100 \mu \mathrm{M}$ substrate in $50 \mathrm{mM}$ sodium citrate buffer ( $\mathrm{pH}$ 6.0) for LOX1:Md:1a and Gly567Ala or $50 \mathrm{mM}$ sodium phosphate buffer ( $\mathrm{pH} 7.5)$ for LOX1:Md:1c, respectively. Each reaction was run in triplicate. The first order kinetic rates were estimated as changes in absorbance at $234 \mathrm{~nm} \mathrm{~s}^{-1}$ and converted into $\mu \mathrm{mol} \mathrm{min}{ }^{-1} \mathrm{mg}^{-1}$, using the extinction coefficient $e=23000 \mathrm{M}^{-1} \mathrm{~cm}^{-1}$ for LA and LnA hydroperoxides. One unit of LOX activity is defined as the amount of enzyme catalysing the formation of $1 \mu \mathrm{mol}$ of hydroperoxide per minute under optimal reaction conditions. The data were then fitted using Michaelis-Menten equation to determine the kinetic parameters for all enzymes.

\section{Identification of LOX products}

Hydroxy fatty acids were separated by straight phase-HPLC (SP-HPLC) using a LiChrospher ${ }^{\circledR} 100$ Diol column (Merck, Darmstadt, Germany; $125 \times 4$ mm $5 \mu \mathrm{m}$ particle size). The solvent used was hexane/2-propanol/formic acid (100:2:0.1, by vol.) and a flow-rate of $2 \mathrm{~mL} \mathrm{~min}^{-1}$, with detection at $234 \mathrm{~nm}$. Fractions of the 9- and 13-enantiomers were collected and submitted to Chiral phase-HPLC (CP-HPLC) for analysis of product stereochemistry. This was carried out on a Chiralpak ${ }^{\circledR}$ IA column (Diacel Chem. Industries, distributed by Altmann Analytik (München, Germany); $250 \times 4.6 \mathrm{~mm}, 5 \mu \mathrm{m}$ particle size) with a solvent system of hexane/ethanol/acetic acid (95:5:0.02, by vol.) and a flow-rate of $0.75 \mathrm{~mL} \mathrm{~min}^{-1}$. The enantiomers of 9- and 13-HODE as well as 15(S)-, 12(S)- and 5(S)-HETE were identified by coelution with authentic standard. 9- and 13-HOTE as well as 11-, 9- and 8-HETE products were identified via their mass and fragmentation pattern in LC-MS analysis based on data for fatty acyl standards derived from the LIPID MAPS Lipidomics Gateway (http://www.lipidmaps.org/data/standards/index.html). LC-MS analysis was performed as described by Huang et al. ${ }^{32}$ The HPLC system consisted of a quaternary pump and a variable wavelength detector, all from Agilent 1100 (Bruker Daltonics, Bremen, Germany). The column was a Luna ${ }^{\circledR}$ C18 (Phenomenex, Aschaffenburg, Germany; $150 \times 2$ mm, $100 \AA, 5 \mu \mathrm{m}$ particle size). Absorbances were recorded at $234 \mathrm{~nm}$ for the detection of hydroxy fatty acids.

\section{Site-directed mutagenesis of MdLOX1a}

Two approaches were chosen to decide on amino-acid positions for sitedirected mutagenesis: (i) the available literature was searched for mutagenesis studies on single amino acids of plant and mammalian LOXs. The primary sequences of characterized enzymes were aligned to LOX1:Md:1a using Geneious, version 5.6.6 (Biomatters; http://www.geneious.com/) to identify corresponding positions in the apple LOX. Amino acids, which are likely to be responsible for stereo- or regiospecificity in LOX, were chosen for mutagenesis; and (ii) homology models of apple LOXs were created by the SWISSMODEL server ${ }^{34}$ using the X-ray coordinates of soybean L-3 (PDB ID 1IK3) as template. Superposed structures of the dual positional specific LOX1:Md:1a and a closely related 9-LOX (LOX1:Md:1c) were visualized with the PyMOL Molecular Graphics System, version 1.3 (Schrödinger, LLC; http://www.pymol.org) and inspected for substitutions in the active site region. Each of these positions was mutated separately in the MdLOX1a gene.

Site-directed mutagenesis was carried out by using the methods described by Horton ${ }^{35}$ or the QuikChange ${ }^{T M}$ Site-Directed Mutagenesis Kit (Stratagene, Amsterdam, The Netherlands). The mutants Arg268Ala, Ile566Phe and Val582Phe were created using overlap extension PCR (oePCR) producing overlapping gene fragments with the desired mutation. In order to minimize the risk of hairpin and self-dimer formation the applied oligonucleotides contained additional conservative base exchanges apart from the appropriate mutations. The mutants Gly567Ala, Leu572lle,
Ile578Leu and Thr775Leu were created following the instructions of the QuikChange ${ }^{\mathrm{TM}}$ protocol. All oligonucleotides used in this experiment are depicted in the Supplementary Table S6. Success of mutagenesis was confirmed by sequencing with gene-specific primers.

Transient expression of yellow fluorescent protein (YFP) fusions in tobacco plants

Subcellular localization of deduced LOX proteins was predicted through the identification of signal peptide sequences using several prediction programs such as ProtComp (http://www.softberry.com/berry.phtml), PSORT (psort.nibb.ac.jp/). ${ }^{36}$ TargetP (http://www.cbs.dtu.dk/services/TargetP/), ChloroP (http://www.cbs.dtu.dk/services/ChloroP/), Signal-3L (http:// www.csbio.sjtu.edu.cn/bioinf/Signal-3L/) and Predotar (http://urgi.versailles.inra.fr/predotar/predotar.html). ${ }^{37}$ Chimeric YFP constructs were produced for MdLOX2a, MdLOX4a and MDLOX6b in the 13-LOX group and MdLOX1a, MdLOX7a and MdLOX7c in the 9-LOX group. All were designed to express the first 166-300 amino-acid residues of the protein including, where present, the putative chloroplast transit peptide. ${ }^{38}$ The YFP sequence was fused to the C-terminus of each LOX sequence. Phusion ${ }^{\circledR}$ High-Fidelity DNA polymerase (New England Biolabs, Arlington, VA, USA) was used for PCR and cloning applications. Directional cloning was performed using the P-ENTR/ D-TOPO entry vector kit (Life Technologies, Carlsbad, CA, USA). After sequencing, purified plasmids were digested with Mlu-I restriction enzyme (New England Biolabs) and a LR clonase recombination reaction between the entry clone and the pEarleyGate 101destination vector (35S-Gateway-YFPHA tag-OCS 3', ABRC stock no. CD3-683 at The Ohio State University) ${ }^{39}$ was performed as described by the manufacturer (Life Technologies). Tobacco (Nicotiana tabacum cv Petit havana) plants, grown for five weeks at $22{ }^{\circ} \mathrm{C}$ in a greenhouse, were used for Agrobacterium tumefaciens-mediated transient expression. Briefly, each expression vector was introduced into Agrobacterium tumefaciens strain GV3101 by heat shock. A single transformed colony was used to inoculate $3 \mathrm{~mL}$ of LB medium containing kanamycin $\left(50 \mu \mathrm{g} \mathrm{m}^{-1}\right)$, gentamycin $\left(50 \mu \mathrm{g} \mathrm{mL}^{-1}\right)$ and rifampicin $\left(10 \mu \mathrm{g} \mathrm{mL}^{-1}\right)$. The bacterial culture was incubated at $28{ }^{\circ} \mathrm{C}$ with agitation for $16 \mathrm{~h}$. An aliquot $(0.2 \mathrm{~mL})$ of bacterial culture then was pelleted in a $1.5-\mathrm{mL}$ Eppendorf tube by centrifugation at $4000 g_{n}$ for $5 \mathrm{~min}$ at room temperature. The pellet was resuspended in $0.5 \mathrm{~mL}$ of infiltration medium (50 mM MES, pH 5.6, $2 \mathrm{mM} \mathrm{NaH}_{2} \mathrm{PO}_{4}, 200 \mu \mathrm{M}$ aceto-syringone and $5 \mathrm{mg} \mathrm{mL}^{-1}$ glucose). The bacterial suspension was diluted with infiltration buffer to adjust the inoculum concentration to a final $\mathrm{OD}_{600}$ value range of $0.05-0.1$. The bacterial suspension was infiltrated using a $1-\mathrm{mL}$ syringe without a needle by gentle pressure through the stomata on the abaxial surface. Transformed plants then were incubated under normal growth conditions for $72 \mathrm{~h}^{40,41}$ Confocal imaging was performed using an inverted laser scanning microscope (IX81 model FV1000; Olympus, Melville, NY, USA) with $\times 20$ and $\times 40$ air objectives. Excised plant tissues were mounted in water between a microscope slide and cover slip and examined. To detect EYFP fluorescence, an argon ion laser was used to produce excitation at a wavelength of $488 \mathrm{~nm}$ and florescence was detected in the range of 505-530 nm. Chlorophyll fluorescence was detected in the range of $670-730 \mathrm{~nm}$ using an excitation laser with a wavelength of $633 \mathrm{~nm}$. All images were acquired from single optical sections. Post-acquisition image processing was done using Olympus Fluoview FV1000 software.

Sequence data: The nucleotide sequences reported in this paper are annotated in the GenBank database at NCBI (http://www.ncbi.nlm.nih.gov) under the accession numbers KC706480 MdLOX1a, KC706481 MdLOX1C, KC706482 MdLOX1d, KC706483 MdLOX2a and KC706485 MdLOX2b.

\section{RESULTS}

Cloning and sequence analysis of LOXs from apple

The oligonucleotides for amplification of MdLOX1 and MdLOX2 genes were designed based on manually curated Malus $\times$ domestica predicted protein sequences. ${ }^{24}$ The MdLOX1 gene cluster comprises five putative functional LOX sequences, whereas the MdLOX2 gene cluster seemed to consist of only two LOX isoforms. Six candidate genes (MdLOX1a, MdLOX1b, MdLOX1c, MdLOX1d, MdLOX2a and MdLOX2b) contained all functional motifs of plant LOX enzymes and were chosen for primer design (Supplementary Table S1). The bioinformatically predicted open reading frame of MdLOX1e (MDP0000312394) exhibited large sequence deletions and lacked the stop codon immediately downstream of the highly conserved 
C-terminal motif. ${ }^{42}$ Also, manual evaluation of the Golden Delicious genome did not succeed in a functional full-length $L O X$ sequence for MdLOX1e.

The PCR amplification of LOX genes on apple-derived CDNA resulted in the successful cloning of three members of the MdLOX1 gene cluster (MdLOX1a, MdLOX1c and MdLOX1d) and both genes of the MdLOX2 group. Nucleotide sequences of MdLOX1a, MdLOX1c and MdLOX1d share an identity of $76 \%-86 \%$ and display highest similarity to the almond PdLOX1 gene (AJ404331) and the hazelnut CaLOX1 gene (AJ417975) with up to $87 \%$ and $79 \%$, respectively (Supplementary Figure $S 1$ ). Both $L O X$ genes have been categorized as type-1 9-LOX. ${ }^{43,44}$ MdLOX2a and MdLOX2b share an identity of $93 \%$ and exhibit highest similarity towards the grapevine VvLOXO gene (71\%, FJ858257), which encodes a functional type-2 13 -LOX. ${ }^{18}$ Sequences of apple LOX genes were submitted to the GenBank database at NCBI (http://www.ncbi.nlm.nih.gov, accession numbers KC706480 MdLOX1a, KC706481 MdLOX1c, KC706482 MdLOX1d, KC706483 MdLOX2a and KC706485 MdLOX2b). The nomenclature of the deduced LOX proteins was employed as described by Vogt et al. ${ }^{24}$ Thus, the resulting protein of the MdLOX2a gene which is a type-2 LOX was named LOX2:Md:2a.

For comparison of protein primary structures, we created a multiple sequence alignment of the deduced amino-acid sequences of all five candidate genes (Supplementary Figure S2). The predicted sizes of LOX2:Md:2a (906 amino acids) and LOX2:Md:2b (905 amino acids) (Supplementary Table S1) were larger compared to the MdLOX1 group, except of LOX1:Md:1d, due to the presence of a putative chloroplast transit peptide, thus designating them as type2 LOX. The deduced proteins LOX1:Md:1a and LOX1:Md:1c lacked an amino-terminal extension and were therefore classified as type-1
LOX. Earlier attempts to predict sub-cellular localization of LOX1:Md:1d has not been unequivocally possible since the gene encodes for an unusually long $\mathrm{N}$-terminus harboring a potential signal peptide. ${ }^{24}$ However, due to the high sequence similarity to genes of the MdLOX1, MdLOX3 and MdLOX7 clusters, it was designated as type-1 LOX.

Furthermore, all MdLOX1 proteins exhibit the TV motif in Sloane position (Supplementary Figure S2; LOX1:Md:1a Thr581/Val582; LOX1:Md:1c Thr580/Val581; LOX1:Md:1d Thr670/Val671) that is indicative of LOX enzymes with 9-LOX function. ${ }^{45,46}$ Whereas both members of the MdLOX2 group harbor the more space filling amino-acid cysteine and phenylalanine typical for linoleate 13LOX (LOX2:Md:2a Cys623/Phe624; LOX2:Md:2b Cys622,/Phe623). In addition, stereospecificity of LOX enzymes is determined by a conserved alanine residue in close proximity to this motif. ${ }^{47}$ All characterized apple LOX proteins possess an alanine at this position (LOX1:Md:1c Ala566; LOX1:Md:1d Ala656; LOX2:Md:2a Ala609; LOX2:Md:2b Ala608) suggesting either 9(S)- or 13(S)-LOX activity. Only LOX1:Md:1 a exhibits a glycine residue (Gly567) responsible for dual positional specificity and (R)-LOX activity. ${ }^{48,49}$

Ethylene, respiration rate and aroma volatiles

To link changes in gene expression with fruit ripening and the concomitant synthesis of hexyl esters (i.e., esters derived from hexanol), we determined progressive changes in the internal ethylene concentration, respiration and ester formation (Figure 2). Eight developmental stages were discernible based on changes in these three indices. Hexyl ester (the sum of hexyl acetate, hexyl propanoate, hexyl butanoate, hexyl pentanoate, hexyl hexanoate, hexyl 2methylpropanoate and hexyl 2-methylbutanoate) emissions were

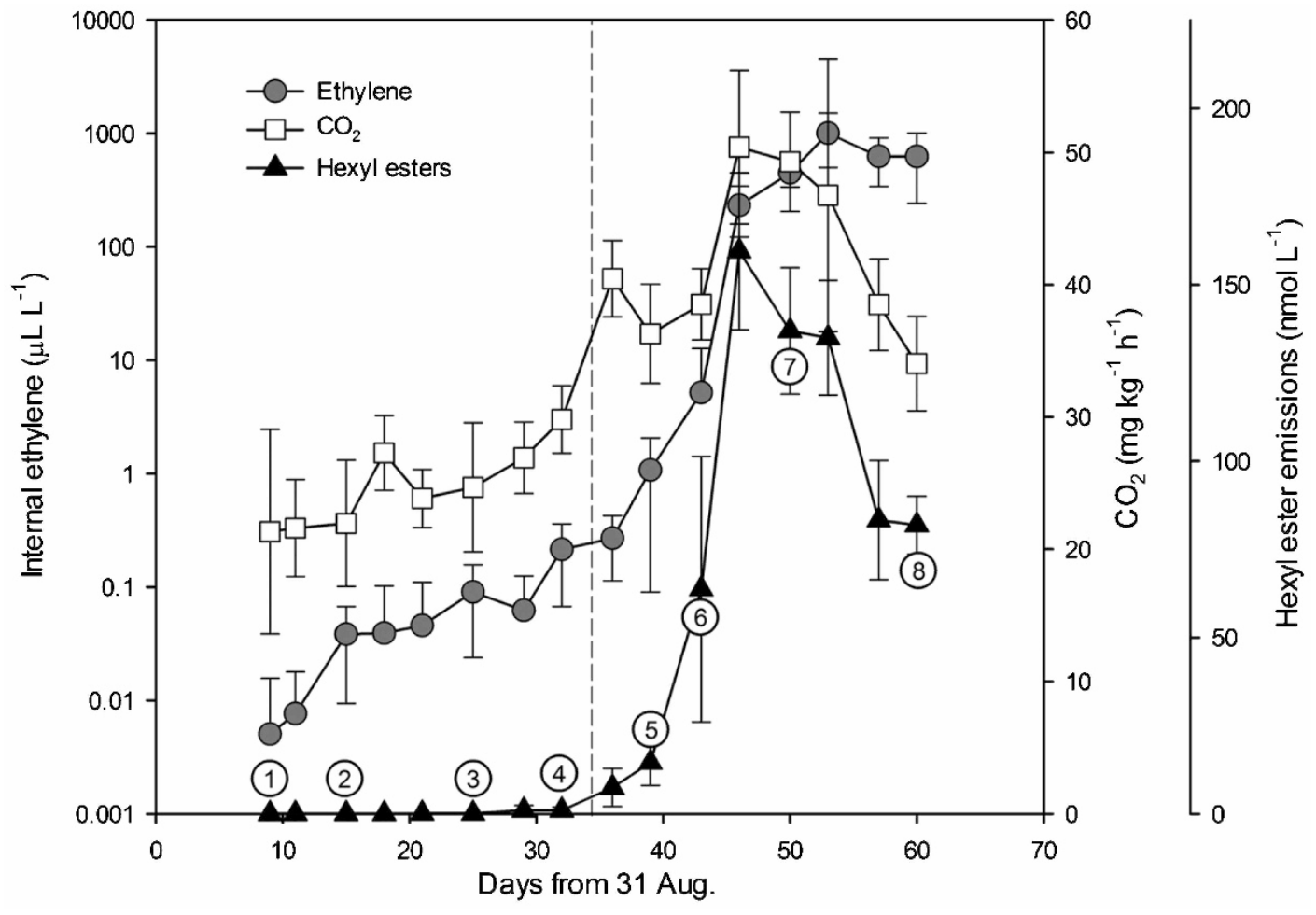

Figure 2. Fruit ripening. Hexyl esters, internal ethylene, and $\mathrm{CO}_{2}$ production during fruit ripening in 'Jonagold' apples. Fruit were assessed from 9 September 2009 (stage 1) to 27 October 2009 (stage 8). Eight stages were identified: immature apple (stage 1), mature with low levels of ethylene (stage 2), mature, low levels of ethylene just prior to the detection of hexyl esters (stage 3), mature/ripening with low but increasing levels of ethylene and low levels of hexyl esters (stage 4), ripening, autocatalytic ethylene synthesis engaged and rapidly increasing ester emissions (stage 5), ripening, at the peak of the respiratory climacteric (stage 6), ripe, at the peak of ester emissions and the onset of the decline in respiration (stage 7) and overripe/senescent with declining ester synthesis and respiratory activity (stage 8 ). 
first detected after stage 3, while internal ethylene levels were low (less than $0.1 \mu \mathrm{L} \mathrm{L}^{-1}$ ), but steadily increasing. The onset of autocatalytic ethylene occurred after stage 4 and coincided with the onset of climacteric respiration and a rapid increase the emissions of hexyl esters.

RT-PCR and real-time qPCR assays

Semi-quantitative RT-PCR of 22 putative $L O X$ genes revealed that 17 were expressed in apple peel (Supplementary Figure S3). The expression of most genes exhibited no discernable pattern during ripening; however, at least six were highly expressed and ripeningdependent. In the putative 13-LOX group, MdLOX4a showed a marked peak at developmental stage 5 , which coincided with the rise in autocatalytic ethylene. The expression of MdLOX6a and MdLOX $6 b$ declined during ripening. In the putative 9-LOX group, MdLOX3a expression declined during ripening, while MdLOX7a and MdLOX1a increased (Supplementary Figure S3). No visible PCR bands were detected from MdLOX1d, MdLOX4a, MdLOX5d, MdLOX5e and MdLOX9a during fruit development.

qPCR was performed on the 6 LOX genes that showed ripeningdependent patterns, and additionally included MdLOX2a due to its lack of variation during ripening to provide additional support for the accuracy of the RT-PCR results. In each instance, the RT-PCR and QPCR results yielded similar results (Figure 3 ). In the putative 13LOX group, MdLOX2a exhibited little change or a slight decrease in expression during ripening, again $M d L O X 4 a$ evidenced a sharp increase at stage 5, and MdLOX6a and MdLOX6b were downregulated during ripening. Within the putative 9-LOX group, MdLOX7c was downregulated, and MdLOX7a and MdLOX1 $a$ were upregulated as ripening progressed. MdLOX1a expression increased more than 100 -fold throughout the course of ripening, with the initial increase occurring between developmental stages 3 and 4, concomitant with the first detectable emissions of hexyl esters.

Biochemical analysis of four recombinant apple LOX

The full-length open reading frame sequences of MdLOX1a and MdLOX1c (type-1 9-LOX) as well as MdLOX2a and MdLOX2b (type-2 13-LOX) were cloned into the pYES2 vector for heterologous expression in yeast. His-tagged apple LOX proteins were partially purified from protein crude extracts by affinity chromatography. SDS-PAGE analysis revealed proteins with a molecular mass of about $105 \mathrm{kDa}$, in accordance with the predicted values for recombinant LOX1:Md:1a (101.5 kDa), LOX1:Md:1c (101.6 kDa), LOX2:Md:2a (106.5 kDa) and LOX2:Md:2b (106.6 kDa) proteins (Supplementary Figure S4). Both members of the MdLOX1 group exhibited highest enzymatic activity with LA at $45{ }^{\circ} \mathrm{C}$ (Supplementary Figure S5a and S5b). Recombinant LOX1:Md:1a showed a rather narrow optimum at $45{ }^{\circ} \mathrm{C}$ with rapidly decreasing activity at lower or higher temperatures, but high residual activity at $55{ }^{\circ} \mathrm{C}$ (54\%). LOX1:Md:1c displayed high catalytic activity in a broad range of temperatures between 15 and $50{ }^{\circ} \mathrm{C}$, with a sharp decrease above $50{ }^{\circ} \mathrm{C}$. In contrast, LOX2:Md:2a and LOX2:Md:2b had lower optimum temperatures of $35{ }^{\circ} \mathrm{C}$ and $25^{\circ} \mathrm{C}$, respectively (Supplementary Figure S5c and S5d). Both enzymes showed negligible activity at temperatures higher than $45{ }^{\circ} \mathrm{C}$. However, it should be mentioned, that LOX2:Md:2b displayed considerably high activity at $5{ }^{\circ} \mathrm{C}(60 \%)$. The members of the MdLOX1 group (Supplementary Figure S5e and S5f) showed an optimum pH of 6.0 (LOX1:Md:1a) and 7.5 (LOX1:Md:1C). Both enzymes exhibited high activity (>80\%) in a broad $\mathrm{pH}$ range with sharply decreasing relative activity below 5.0 and above 7.5. The members of the MdLOX2 group had an optimum $\mathrm{pH}$ of 7.0. They showed moderate enzyme activity (greater than $60 \%$ of relative activity) in a pH range of $4.5-8.0$ (LOX2:Md:2a) and 6.0-9.5 (LOX2:Md:2b), but only a rather narrow range of high activity around neutral $\mathrm{pH}$ (Supplementary Figure $\mathrm{S5g}$ and S5h).

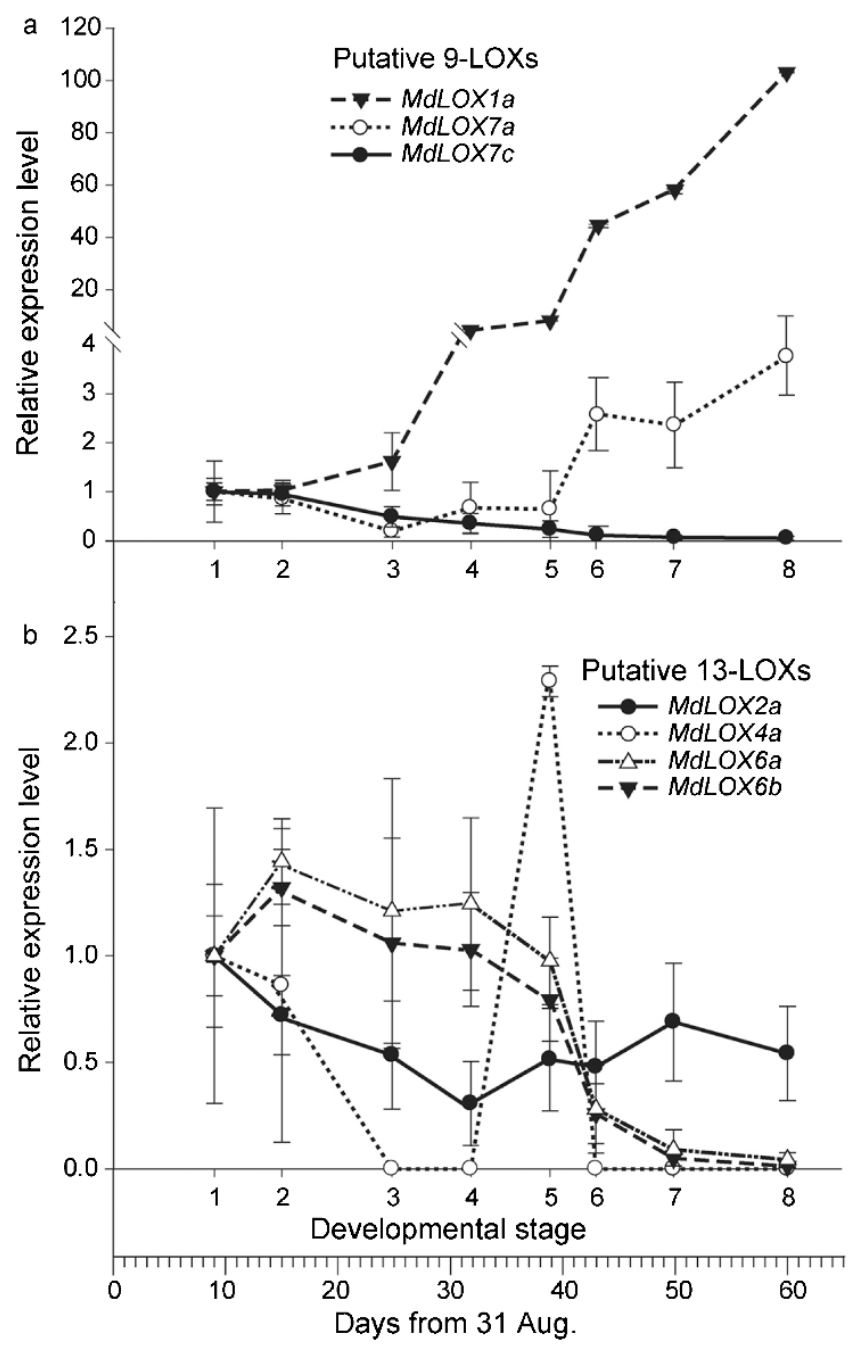

Figure 3. Expression of selected $L O X$ genes. Relative expression levels of putative 9-LOX (upper panel) and 13-LOX (lower panel) transcripts using qPCR for peel tissue from 'Jonagold' apple fruit during ripening. Expression is normalized relative to the earliest (preclimacteric) developmental stage evaluated. Time points selected are the same as those depicted in Figure 2, with the onset of autocatalytic ethylene production occurring between stages 4 and 5 .

Regio- and stereochemistry of reaction products

Reaction products of LA, LnA and arachidonic acid (AA) formed by the four proteins were analyzed to characterize regio- and stereospecificity of the apple LOXs. Partially purified LOX1:Md:1a, LOX1:Md:1c, LOX2:Md:2a and LOX2:Md:2b proteins converted all three tested substrates to their corresponding hydroperoxy fatty acids, as was confirmed by LC-MS analysis (Supplementary Figure S6). Additionally, reaction products with LA were reduced to their corresponding hydroxy fatty acids and analyzed using SP-HPLC (Figure 4). Fractions of 9- and 13-HODE were collected and analyzed for enantiomer composition by CP-HPLC (Figure 4 insets). Both, LOX1:Md:1 $\mathrm{a}$ and LOX1:Md:1c produced 9(S)-HpODE from linoleic acid (Figure 4a and 4b), but LOX1:Md:1 a formed primarily 13(R)HpODE (about 77\% of total hydroperoxides). LOX1:Md:1 a acted as a dual positional specific enzyme producing 13- and 9-HpODE in a ratio of 8:1 (Table 1). In contrast, 9(S)-HpODE was the major product of LOX1:Md:1C activity with LA, as only trace amounts of the regioisomer $(0.5 \%$ of total hydroperoxides) and enantiomer $(2.3 \%)$ was formed. Similar results were obtained for LOX activity of both 

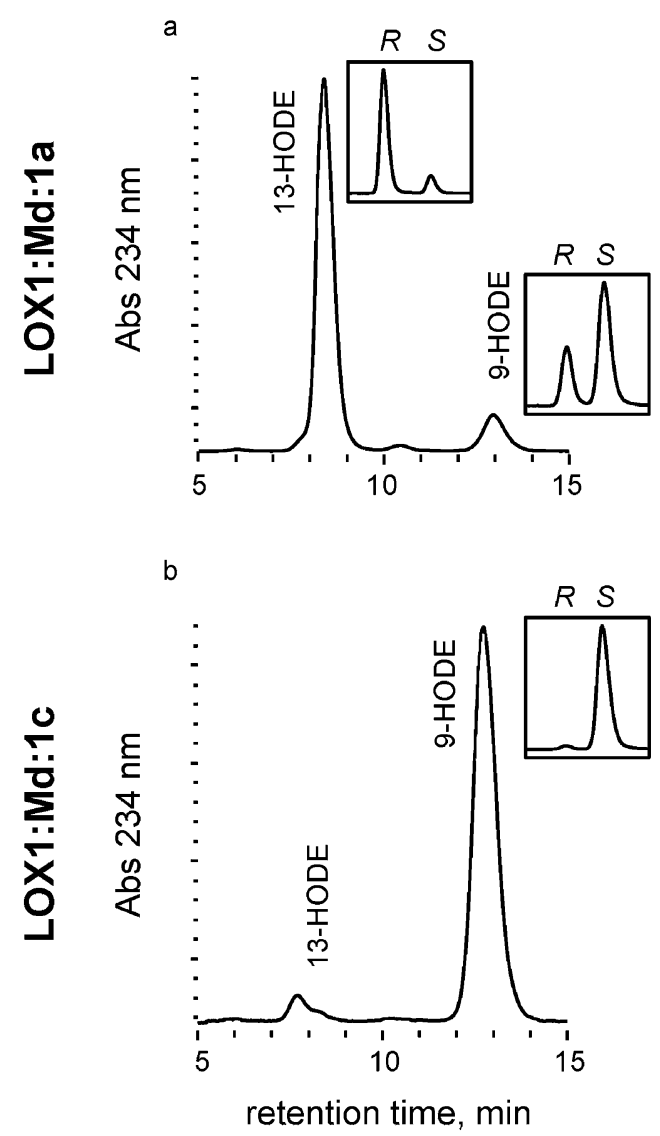
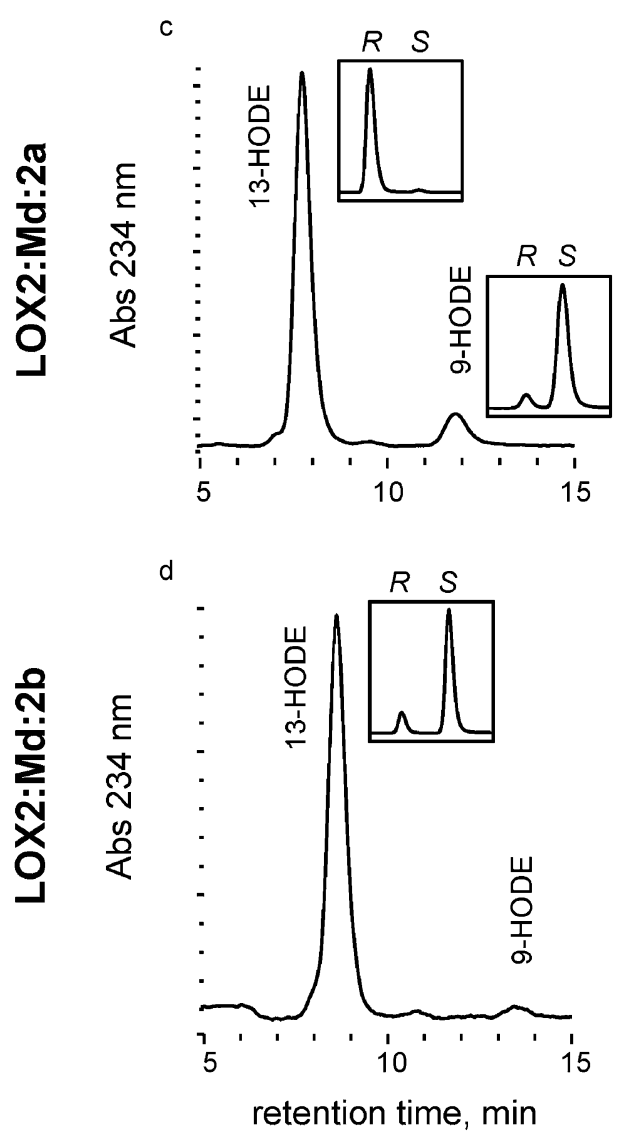

Figure 4. HPLC analysis of hydroxy fatty acids formed by wild-type apple LOX enzymes followed by chemical reduction. Product profiles for LOX activity with linoleic acid were obtained via SP-HPLC. Ratios of $(R)$ - and $(S)$-enantiomers were determined by CP-HPLC (Insets). (a) Wild-type LOX1:Md:1a. (b) Wild-type LOX1:Md:1c. (c) Wild-type LOX2:Md:2a. (d) Wild-type LOX2:Md:2b. A LiChrospher@ 100 Diol column (125×4 mm, $5 \mu \mathrm{m}$ ) was eluted at $2 \mathrm{~mL} \min ^{-1}$ with hexane:2-propanol:formic acid (100:2:0.1, by vol.) with UV detection at $234 \mathrm{~nm}$. Chiral phase HPLC analysis of enantiomer composition was conducted on a Chiralpak ${ }^{\circledR}$ IA column $(250 \times 4.6 \mathrm{~mm}, 5 \mu \mathrm{m})$ with an elution at $0.75 \mathrm{~mL} \mathrm{~min}^{-1}$ in hexane/ ethanol/acetic acid (95:5:0.02, by vol.) and UV detection at $234 \mathrm{~nm}$.

enzymes with LnA (Supplementary Table S4). LOX2:Md:2a and LOX2:Md:2b converted LA and LnA predominantly to 13-hydroperoxides (Figure $4 \mathrm{C}$ and $4 \mathrm{~d}$ ). However, the main product of LOX2:Md:2b reaction with LA was identified as $13(S)-H p O D E$, whereas LOX2:Md:2a formed 13(R)- and 9(S)-HpODE in a ratio comparable to LOX1:Md:1a. Accordingly, LOX1:Md:1a and LOX2:Md:2a

Table 1. Linoleic acid products formed by wild-type apple LOX enzymes. Proportion of hydro(pero)xy fatty acids is given as percent of total product amount obtained from specific LOX activity with linoleic acid

\begin{tabular}{lcc}
\hline Enzyme & 9-H(p)ODE $(\%)$ & 13-H(p)ODE (\%) \\
\hline LOX1:Md:1a & 11.2 & 88.8 \\
LOX1:Md:1c & 99.5 & 0.5 \\
LOX2:Md:2a & 12.4 & 87.6 \\
LOX2:Md:2b & 6.0 & 94.0 \\
\hline
\end{tabular}

\begin{tabular}{lrrrr}
\hline & \multicolumn{5}{c}{ Chiral analysis (\%) } \\
\cline { 2 - 5 } & $9 R$ & $9 S$ & $13 R$ & $13 S$ \\
\hline LOX1:Md:1a & 29.1 & 70.9 & 86.8 & 13.2 \\
LOX1:Md:1c & 2.3 & 97.7 & \multicolumn{2}{c}{ racemic } \\
LOX2:Md:2a & 8.8 & 91.2 & 97.9 & 2.1 \\
LOX2:Md:2b & \multicolumn{2}{c}{ racemic } & 13.6 & 86.4 \\
\hline
\end{tabular}

were classified as $(R)$-LOX with a 13(R)/9(S)-specificity. LOX1:Md:1c and LOX2:Md:2b proved to be (S)-LOX enzymes and were classified as linoleate 9(S)- and 13(S)-LOX, respectively.

Additionally, we tested for substrate conversion of AA by all four apple LOXs. Arachidonic acid is of minor physiological importance in plants and is therefore often a poor substrate for plant LOXs. Even so, all wild-type apple LOX enzymes were able to convert AA at least to some extent to hydroperoxy eicosatetraenoic acid (HpETE). LOX2:Md:2b showed highest product specificity as it formed solely 15-HpETE (Supplementary Table S4). LOX1:Md:1a and LOX2:Md:2a produced considerable amounts of $15-\mathrm{HpETE}(42 \%-45 \%)$, but also 12-HpETE (16\%-17\%), 9-HpETE (12\%-16\%) and small amounts of $11-$, 8- and 5-HpETE from AA. The major AA products formed by LOX1:Md:1c was 11-HpETE (53\%) and 5-HpETE (18\%).

Site-directed mutagenesis of MdLOX1a

To further evaluate the potential for the involvement of LOX1:Md:1a in autonomous aroma formation in ripe apple fruits, we investigated the determinants of LOX1:Md:1a specificity by site-directed mutagenesis of the MdLOX1a gene and subsequent biochemical analysis of the heterologously expressed mutant proteins. To compare our findings on enzymatic activity, we chose LOX1:Md:1c as a reference. Both apple LOXs share a sequence identity of $85 \%$ and a highly conserved active site with the putative 9-LOX-specific TV motif (Supplementary Figure S2, LOX1:Md:1a Thr581/Val582; LOX1:Md:1c Thr580/Val581) at the so-called Sloane position. 
However, only LOX1:Md:1c produced exclusively 9(S)-hydroperoxides from LA and LnA. Comparison of their primary structures revealed four positions that differed in the enzyme active sites of both proteins (Supplementary Figure S2). Thus, Gly567, Leu572, lle578 and Thr775 in LOX1:Md:1a were individually replaced by the corresponding amino acids of LOX1:Md:1c, resulting in the single mutants Gly567Ala, Leu572lle, Ile578Leu and Thr775Leu (Figure 5). Three additional positions were mutated to elucidate the role of an inverse head-to-tail orientation of the substrate in the active site on the production of 13-hydroperoxides by LOX1:Md:1a. ${ }^{46}$ In the straight substrate orientation, free fatty acids enter the hydrophobic cavity with their methyl end first. The carboxyl group is then supposed to form salt bridges with polar residues near the cavity entrance. ${ }^{50}$ In LOX1:Md:1a, the positions Arg268 and Lys 283 were candidates for such a salt bridge formation. Their effect on product regiochemistry was analyzed by substitution of Arg268 with a hydrophobic alanine residue (Figure 5). In addition, lle566 at the cavity entrance was substituted by the more space-filling phenylalanine residue of LOX1:Md:1c to analyze a potential steric hindrance of salt bridge formation. A similar effect should be observed for substitution of Val582 at the Sloane position by a phenylalanine residue. Arg268Ala and Ile566Phe should interfere with the interaction of the carboxyl group and polar residues at the cavity entrance, leading to a preference of inverse substrate orientation and thereby 9(S)-LOX activity, whereas Val582Phe should hinder salt bridge formation of the carboxyl group and the conserved arginine residue (Arg732) at the bottom of the pocket, resulting in a straight substrate orientation and 13(S)-LOX activity. In total, seven positions were chosen for site-directed mutagenesis (Figure 5).

Of these seven analyzed single mutants only Arg268Ala, Gly567Ala, Ile578Leu and Val582Phe showed high substrate conversion rates of $L A$, $L n A$ and $A A(60 \%-100 \%$ of $L O X 1: M d: 1 a$ activity with LA). The Thr775Leu mutant exhibited significant lower activity ( $~ 33 \%$ of LOX1:Md:1a activity with LA), whereas LOX activity of the Ile566Phe and Leu572lle mutants was nearly completely abolished. Hydroperoxy products formed by the different mutant LOX proteins showed regio- and stereospecificity similar to LOX1:Md:1a, except for Gly567Ala (Figure 6). The mutation of Gly567 to alanine affected an amino acid residue, which has been previously described to determine LOX stereoselectivity. ${ }^{47}$ Instead of forming a mixture of $13(R)$ - and $9(S)-H p O D E$, the Gly567Ala mutant protein acted as a strict 9-LOX producing mainly 9(S)-HpODE (about $87.5 \%$ of total hydroperoxides) from linoleic acid (Table 2). Also, reactions of the mutant enzyme with LnA and AA yielded product profiles similar to LOX1:Md:1c (Supplementary Table S4). Mutation of Val582 to the more space-filling phenylalanine did not change regio- or stereospecificity of the enzyme (Figure 6). The Val582Phe mutant still formed $13(R)-\mathrm{HpODE}$ as the major product from LA. However, the low amounts of the $9-\mathrm{HpODE}$ product consisted of a racemic mixture of both enantiomers (Table 2). Product profiles detected for the reaction with $\operatorname{LnA}$ and $A A$ were similar to those observed for wild-type LOX1:Md:1a protein. In contrast, the major products of the lle578Leu mutant with AA were 9-HpETE (29\%) and 5-HpETE (21\%) (Supplementary Table S4). Although the Ile578Leu mutant mainly formed 13-hydroperoxides from LA and LnA, a slight loss of regioselectivity was observed, leading to increased levels of the 9-hydroperoxides.

Since the Gly567Ala mutation completely changed the specificity of hydroperoxide formation, the kinetic properties of the mutant protein was determined and compared to the values of the wildtype LOX1:Md:1a and LOX1:Md:1c. Hydroperoxide formation was monitored across a range of concentrations of LA and LnA. The obtained velocity curves did not fit typical Michaelis-Menten kinetics. Most curves exhibited a more complex pattern with declining enzyme velocity at higher substrate concentrations (Supplementary Figure S7). Therefore, data from the linearly increasing part of the enzyme velocity curves were fitted using the Michaelis-Menten equation. The calculated kinetic parameters for each enzyme were confirmed with Lineweaver-Burk plots (Table 3).

Calculation of the catalytic efficiency of wild-type LOX1:Md:1a revealed only a slight preference for LA $\left(0.26 \mu \mathrm{M}^{-1} \mathrm{~s}^{-1}\right.$ versus $0.19 \mu \mathrm{M}^{-1} \mathrm{~s}^{-1}$ for $\left.\operatorname{LnA}\right)$. However, comparison of the values for substrate affinity $\left(K_{\mathrm{m}}\right)$ and turnover $\left(k_{\mathrm{cat}}\right)$ indicated that while LOX1:Md:1a had a higher affinity for LA (about 2.5-fold higher than for LnA), substrate conversion of LnA occurred twofold faster. Interestingly, mutation of Gly567 to alanine led to a strong enhancement in catalytic efficiency $\left(k_{\text {cat }} / K_{\mathrm{m}}\right)$ for hydroperoxidation of both substrates. The substrate turnover was nearly 12 -fold increased for LA and 4.8-fold increased for LnA. In addition, the mutant had a slightly higher affinity for $\operatorname{LnA}\left(K_{\mathrm{m}}=15.53 \mu \mathrm{M}\right)$ than wild-type LOX1:Md:1a. These results

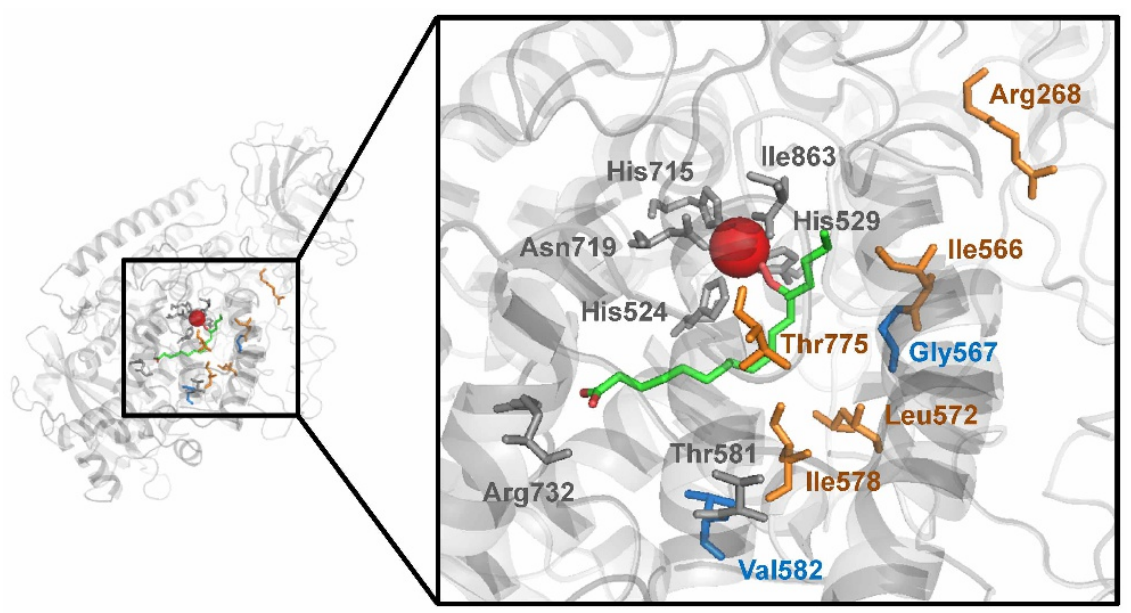

Figure 5. Overview of the substrate binding pocket of LOX1:Md:1a. Homology model showing positions of amino-acid residues described to be essential for LOX activity and specificity (gray, blue) as well as positions chosen for site-directed mutagenesis (orange, blue). A 13(S)-HpODE molecule (green) illustrates position and depth of the substrate binding pocket. The bottom of the cavity is marked by Arg732, which is essential for enzyme activity and therefore highly conserved among plant LOX enzymes. However, the catalytic iron atom (red sphere) is coordinated by five conserved residues (His524, His529, His715, Asn719, Ile863) near the entrance. The residues Thr581 and Val582 align with the so-called Sloane position described for mammalian LOX and are known to control regiospecificity of fatty acid oxygenation. In addition, Gly567 has been described to influence both regio- and stereoselectivity of LOX enzymes. 

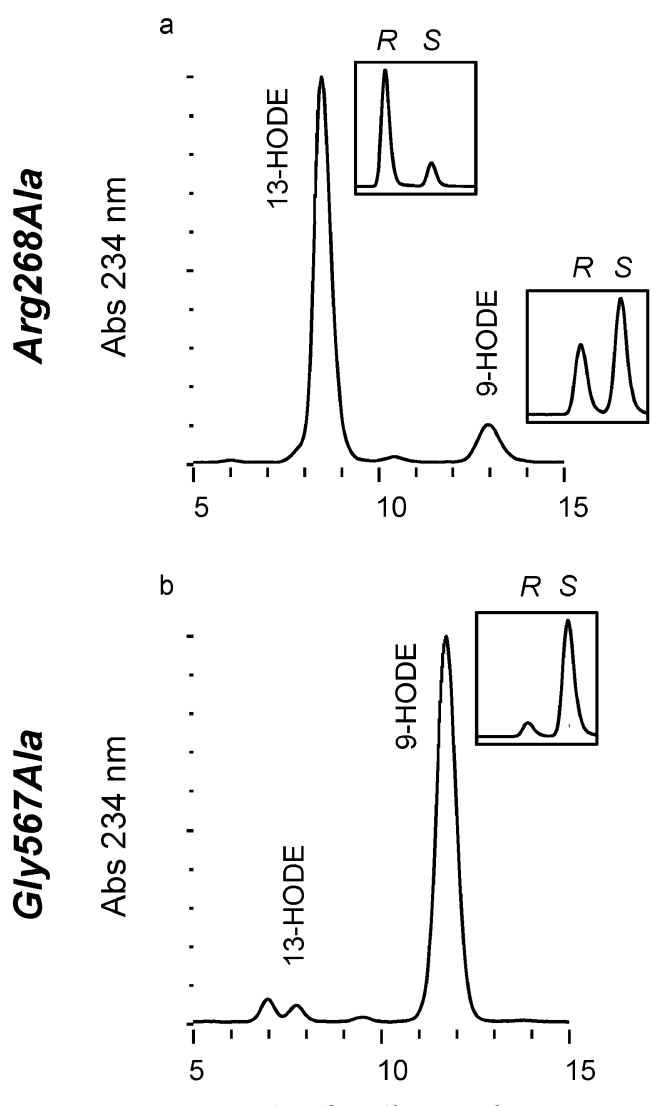

retention time, $\min$
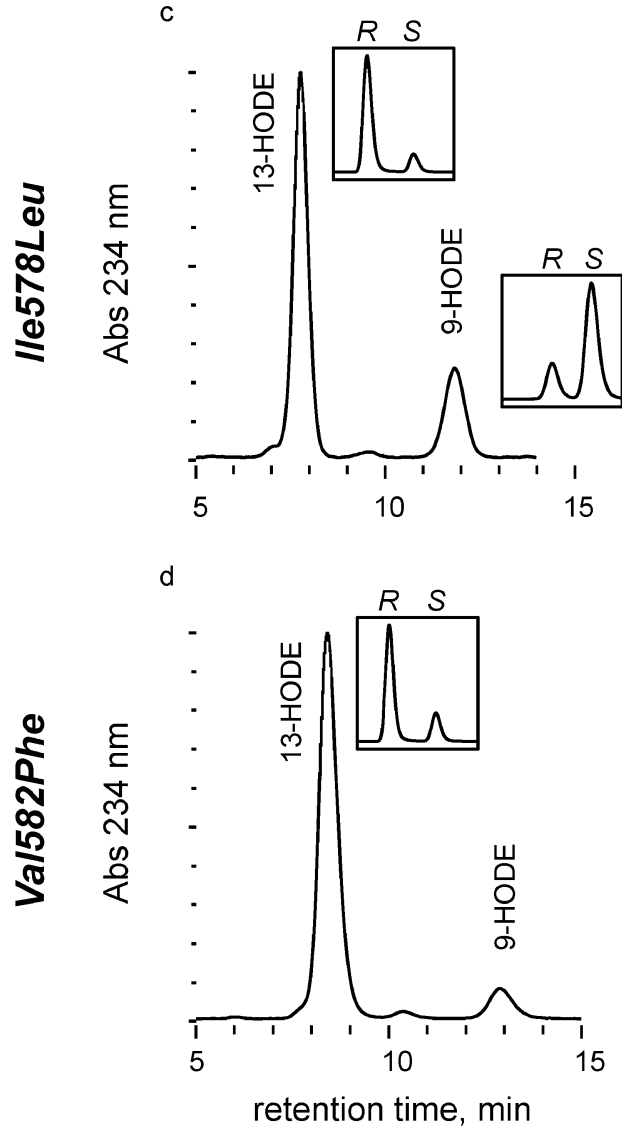

Figure 6. HPLC analysis of hydroxy fatty acids formed by MdLOX1a mutants followed by chemical reduction. Product profiles for LOX activity with linoleic acid were obtained via SP-HPLC. Ratios of (R)- and (S)-enantiomers were determined by CP-HPLC (insets). (a) Arg268Ala mutant. (b) Gly567Ala mutant. (c) Ile578Leu mutant. (d) Val582Phe mutant. A LiChrospher ${ }^{\circledR} 100$ Diol column $(125 \times 4 \mathrm{~mm}, 5 \mu \mathrm{m})$ was eluted at $2 \mathrm{~mL}$ min-1 with hexane/2-propanol/formic acid (100:2:0.1, by vol.) with UV detection at $234 \mathrm{~nm}$. Chiral phase HPLC analysis of enantiomer composition was conducted on a Chiralpak ${ }^{\circledR}$ IA column $(250 \times 4.6 \mathrm{~mm}, 5 \mu \mathrm{m})$ with an elution at $0.75 \mathrm{~mL} \mathrm{~min}^{-1}$ in hexane/ethanol/acetic acid $(95: 5: 0.02$, by vol.) and UV detection at $234 \mathrm{~nm}$.

led to a catalytic efficiency of $2.78 \mu \mathrm{M}^{-1} \mathrm{~s}^{-1}$ for LA- and $1.62 \mu \mathrm{M}^{-1}$ $\mathrm{s}^{-1}$ for LnA-hydroperoxidation and demonstrate that LA is the preferred substrate of the Gly567Ala mutant. The 9(S)-LOX LOX1: Md:1c showed a lower affinity to LA $\left(K_{m}=40.61 \mu M\right)$ and $\operatorname{LnA}$ $\left(K_{\mathrm{m}}=70.85 \mu \mathrm{M}\right)$ than LOX1:Md:1a, but exhibited a higher substrate turnover rate comparable to the Gly567Ala mutant. Also, the catalytic efficiency for LA was 2.8-fold higher compared to LnA.

Table 2. Linoleic acid products formed by MdLOX1a mutants. Proportion of hydro(pero)xy fatty acids is given as percent of total product amount obtained from specific LOX activity with linoleic acid

\begin{tabular}{lcc}
\hline Enzyme & 9-H(p)ODE (\%) & 13-H(p)ODE (\%) \\
\hline Arg268Ala & 10.9 & 89.1 \\
Gly567Ala & 97.3 & 2.7 \\
Ile578Leu & 22.6 & 77.4 \\
Val582Phe & 8.7 & 91.3 \\
\hline
\end{tabular}

\begin{tabular}{lcccc}
\hline & \multicolumn{5}{c}{ Chiral analysis (\%) } \\
\cline { 2 - 5 } & $9 R$ & $9 S$ & $13 R$ & $13 S$ \\
\hline Arg268Ala & 37.5 & 62.5 & 82.5 & 17.5 \\
Gly567Ala & 10.1 & 89.9 & \multicolumn{2}{c}{ racemic } \\
Ile578Leu & 22.2 & 77.8 & 86.5 & 13.5 \\
Val582Phe & \multicolumn{2}{c}{ racemic } & 79.1 & 20.9 \\
\hline
\end{tabular}

\section{LOX protein localization}

Selected LOX genes were evaluated for their association with chloroplasts in a heterologous system using Agrobacterium-mediated transient expression in tobacco. Clear differences between the patterns of protein accumulation were observed among the six lipoxygenases studied (Supplementary Figure S8). Of the three members of the 13-LOX group evaluated, each had putative chloroplast transit peptides. However, only MdLOX4a and MdLOX6b co-located with chloroplasts, but MdLOX2a did not. The observed punctate pattern has been seen for other LOXs and also in genes that participate downstream of the LOX pathway (HPL, AOS, AOC). ${ }^{51}$ None of the three members of the 9-LOX group had putative chloroplast targeting peptides and, consistent with this, none were found to colocate with chloroplasts in the tobacco system.

\section{DISCUSSION}

The $\mathrm{C} 6$ volatiles such as hexanal, Z-3-hexenal, E-2-hexenal and Z-3hexenol are important for the characteristic aroma of different fruit products and are formed by LOX action and subsequent enzymatic reactions during maceration of leaf and fruit tissue. The unsaturated alcohols and aldehydes are abundant in the headspace or extracts of fruit juice or pulped tissue, whereas saturated esters predominate in the headspace volatiles above intact fruits such as apples. ${ }^{8,52}$ In most plant species (except tomato), aldehydes are more abundant in unripe fruit and esters in ripe fruit as esters 
Table 3. Kinetic parameters of purified wild-type apple LOX and the Gly567Ala mutant of MdLOX1a

\begin{tabular}{|c|c|c|c|c|c|}
\hline & \multirow[b]{2}{*}{ Substrate } & \multirow{2}{*}{$\frac{K_{\mathrm{m}}}{(\mu \mathrm{M})}$} & \multirow{2}{*}{$\frac{V_{\max }}{\left(\mu \mathrm{mol} \min ^{-1} \mathrm{mg}^{-1}\right)}$} & \multirow{2}{*}{$\frac{k_{\text {cat }}}{\left(\mathrm{s}^{-1}\right)}$} & \multirow{2}{*}{$\frac{k_{\text {cat }} / K_{\mathrm{m}}}{\left(\mu \mathrm{M}^{-1} \mathrm{~s}^{-1}\right)}$} \\
\hline & & & & & \\
\hline LOX1:Md:1a & LA & $10.35 \pm 0.28$ & $1.57 \pm 0.07$ & $2.67 \pm 0.13$ & 0.26 \\
\hline LOX1:Md:1c & LA & $40.61 \pm 3.10$ & $30.21 \pm 0.93$ & $51.14 \pm 1.58$ & 1.26 \\
\hline & $\operatorname{Ln} A$ & $70.85 \pm 5.48$ & $18.22 \pm 2.69$ & $30.86 \pm 4.56$ & 0.44 \\
\hline Gly567Ala & LA & $11.43 \pm 1.21$ & $18.74 \pm 0.86$ & $31.75 \pm 1.45$ & 2.78 \\
\hline
\end{tabular}

accumulate when the fruit undergoes a climacteric rise in ethylene production. ${ }^{53}$ Thus, in apples and other fruits, most of the studies on aroma formation have focused on the last step of ester formation, in which AAT enzymes have a key role. ${ }^{54}$ Ester biosynthesis in apple is also limited by the levels of alcohols, which implies that a critical step for ester production may be regulated at the level of $A D H$ and LOX.52,55,56 In the following, we provide strong evidence that LOX1:Md:1a is involved in the formation of C6 precursor substrates for AAT and is essential for ester production during climacteric ripening of apples whereas LOX1:Md:1C, LOX2:Md:2a and LOX2:Md:2b probably contribute to $\mathrm{C} 6$ aldehyde production in immature fruit after decompartmentation.

MdLOX1a is highly expressed during final fruit ripening after picking Initially, the involvement of the LOX pathway in the generation of fruit esters was discovered by feeding experiments using unlabeled and isotopically labeled precursors. ${ }^{8}$ Although $\beta$-oxidation of fatty acids has been established as one of the primary processes contributing to the pool of alcohols and acyl-CoA for ester production, unsaturated straight-chain esters appear to arise only by the LOX pathway. Besides, precursors for butyl and possibly also propyl and pentyl ester may be formed from unsaturated fatty acids by sequential activity of LOX/HPL followed by $\beta$-oxidation. As ester composition and levels differed in apples treated with different signal molecules such as salicylic acid, ethephon (ethylene precursor) and methyl jasmonate, it was supposed that regulation of the pathway upstream of AAT activity depends on the regulation of LOX and ADH activity by the signal molecules. ${ }^{57}$ Consistent with this, the suppression of ethylene biosynthesis ${ }^{58}$ and perception ${ }^{59}$ in apple caused a major reduction in all group of volatiles, especially in esters and alcohols. Exposure of apple fruit (in which ethylene synthesis had been suppressed) to ethylene led to a recovery in the levels of alcohols and aldehydes. ${ }^{58}$ While AAT enzyme activity displayed a clear pattern concomitant with ethylene regulation, total LOX enzyme activity (mixture of all LOX) seems to be independent of ethylene modulation. ${ }^{58}$ However, MdLOX1a is the only LOX gene that underwent a sustained increase in expression level concomitant with the onset of the rise in ripening-related ethylene and the climacteric rise in $\mathrm{CO}_{2}$ production and hexyl ester formation. Given that no Z-3-hexenal, Z-3-hexenol or esters derived from Z-3-hexenal are detected in emissions from intact fruit, ${ }^{7}$ LOX metabolism of fatty acid substrates is apparently limited to LA in intact tissues, which would yield hexanal, and subsequently, hexanol and hexyl esters. This conclusion is consistent with the observations that free $\operatorname{Ln} A$ levels are quite low in ripening apple fruit, while LA levels are substantially higher. ${ }^{60}$ Given the low expression of MdLOX1a in immature fruit, other LOX genes are more likely to contribute to C6 aldehyde synthesis prior to ripening.

Contributions of 13-LOX family members to aldehyde production in disrupted tissue

Since at least 17 LOX genes were expressed during different stages of apple fruit ripening (Supplementary Figure S8 and Ref. 24), most of which changed little, the overall LOX enzymatic activity probably remains relatively stable throughout ripening. Even so, it is interesting to consider that only MdLOX6a and MdLOX6b had expression profiles that matched the ripening-related decline in synthesis of $Z$ 3-hexenal reported for disrupted apple tissue. ${ }^{7}$ This and the colocalization of the protein for MdLOX6b with chloroplasts, which are typically rich in $\operatorname{LnA}{ }^{61,62}$ are suggestive of a link between the expression of MdLOX $6 b$ and Z-3-hexenal formation in both immature and ripening apple. In that only one of five LOX enzymes in tomato has been demonstrated to contribute to aldehyde formation by disrupted tomato tissue, ${ }^{16}$ it may be that a limited number of 13-LOX enzymes contribute significantly to the formation of C6 aldehydes from disrupted apple tissue. Intriguingly, the LOX essential for aroma formation in tomato, TomloxC, is a 13-LOX localized to the chloroplast, ${ }^{16}$ much the same as MdLOX6b. Furthermore, the $\mathrm{pH}$ of the juice of disrupted apple tissue, which is typically near $3.5,{ }^{63}$ would be expected to differentially affect LOX enzyme activity. Based on the pH-dependent activity profiles demonstrated herein, LOX2:Md:2b activity would be completely suppressed, for instance, while that of the other three LOX enzymes would not.

MdLOX $1 a$ is associated with a quantitative trait locus cluster for ester-type volatiles

Large-scale statistical analysis of apple EST libraries revealed an apple LOX gene (EST cluster MD187410) as potentially important in volatile ester formation in ripening fruit. ${ }^{25}$ Transcripts of this $L O X$ gene was barely detectable at 123 days after full bloom (DAFB), increased in abundance before the onset of significant volatile production, peaked at 162 DAFB and remained at high levels 183 DAFB. This gene was later mapped on the apple linkage group 9 closely to a quantitative trait locus cluster for volatile esters such as hexyl acetate $^{26}$ and is identical with MdLOX1a. However, in contrast to an expected 13-LOX activity, detailed in silico analysis of the encoded protein sequence classified LOX1:Md:1a as putative 9LOX due to a TV motif in the product specificity site. Therefore, biochemical characterization of the encoded proteins of ripeningrelated apple LOX genes (MdLOX1a, $2 a$ and $2 b$ ) and of LOX1:Md:1c was performed.

LOX1:Md:1a is a dual specific lipoxygenase

The first LOX enzyme isolated from apple fruit was a membranebound protein, which preferably converted LA to hydroperoxy products. $^{64}$ This enzyme displayed optimal activity at pH 6.9 and reacted to changing $\mathrm{pH}$ values similar to LOX2:Md:2a. Another purified apple LOX was found to exhibit a pH optimum of 6.0 and preferably converted linolenic acid. ${ }^{65}$ The product mainly consisted of the 13-hydroperoxide, which was in agreement with LOX activity found in apple homogenates. ${ }^{66,67}$ Chiral analysis revealed $13(R)$ HODE, 9(S)-HODE and their ketones as major metabolites of total LOX activity with LA in stored apple fruits. ${ }^{67}$ Regio- and stereochemistry of the reported metabolites showed a striking similarity to the products gained from the LOX1:Md:1a and LOX2:Md:2a reaction with LA. Therefore, we propose a significant contribution of LOX2:Md:2a and LOX1:Md:1a to hydroperoxide formation during 


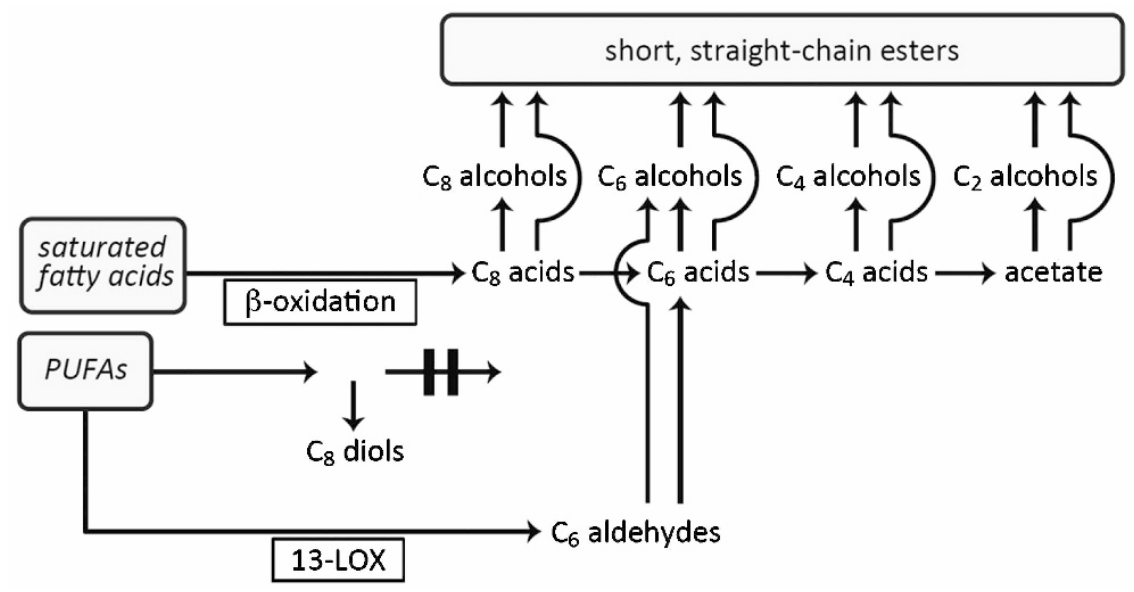

Figure 7. Proposed metabolism of fatty acids in the intact apple fruit. Saturated and mono-saturated fatty acids are degraded to precursor molecules for straight-chain esters via $\beta$-oxidation. For PUFAs, this process seems to be partially impaired. Precursors for straight chain esters might be formed via the action of the LOX pathway, instead. PUFA, polyunsaturated fatty acid.

the early and late stages of fruit ripening, respectively in accordance with the corresponding gene expression pattern. ${ }^{24}$

In our study, three out of four characterized apple LOX were able to form 13-hydroperoxides from LA and LnA. The major fraction of 13-HpODE detected for LOX2:Md:2b was in (S)-configuration, whereas LOX1:Md:1a and LOX2:Md:2a formed mainly 13(R)HpODE. In contrast to the in silico prediction, LOX1:Md:1a was identified as $(R)$-specific dual positional LOX. $(R)$-LOX specificity is a rare trait among plant LOXs and has so far only been described for type-2 LOX from soybean and pea as well as an olive 9/13-LOX. ${ }^{68,69}$ Similar to LOX1:Md:1a, olive 9/13-LOX harbors a glycine residue (Gly568) in the sequence position determining stereoselectivity of fatty acid oxygenation. In contrast, LOX2:Md:2a harbors alanine (Ala609), which is conserved among (S)-LOXs. ${ }^{47}$ It has been suggested that type-2 LOXs with an alanine residue are able to mimic $(R)$-LOX specificity depending on varying substrate concentrations and $\mathrm{pH}^{48}$ However, taking recent theories on stereo- and regiospecificity of LOXs into account, this would lead to the formation of $9(R)$ - and 13(S)-hydroperoxides. Based on the current knowledge it is difficult to predict which mode of substrate binding into the active site would cause the unexpected product specificity of LOX2:Md:2a. Factors that determine regio- and stereospecificity of LOX1:Md:1a are discussed in Supplementary Figure S9.

Furthermore, HPLs have been shown to exhibit a high degree of substrate specificity. ${ }^{70}$ Most HPLs specifically convert either 9(S)- or 13(S)-hydroperoxides of LA and LnA, or both. ${ }^{71,72}$ This, however, raises the question, how $13(R)$-hydroperoxides are metabolized in apple fruits. There is only limited information available on HPL from apple. The enzymatic conversion of $L A$ and $\operatorname{Ln} A$, respectively, to hexanal and $E$-2-hexenal has been reported in homogenates prepared from apple fruits. ${ }^{73}$ Later an enzyme with HPL activity was partially purified and shown to convert 13-hydroperoxides to hexanal, E-2-hexenal and Z-3-hexenal, whereas 9-hydroperoxides were not accepted as potential substrates. ${ }^{74}$

MdLOX1a contributes to aroma formation in apples

The typical flavor profile of an intact apple fruit, which consists mainly of short-chain esters, develops during ripening with maximum volatile formation near the peak of the respiratory climacteric and the rise in ethylene production. Aldehydes that are produced at only low amounts in intact fruits are formed in copious amounts by the action of LOX enzymes upon cell disruption. ${ }^{7}$ While LOX enzymes are present in intact systems prior to disruption, it is not known if LOX contribute to the synthesis of aroma active compounds ( 66 aldehydes and esters) in non-disrupted tissue, although its involvement has been proposed. ${ }^{8}$

It is evident that all four analyzed apple LOXs are catalytically active and reaction specificity as well as developmental changes in gene expression suggest in vivo functions for each isoenzyme during apple ripening. Likewise, a shift in LOX pathway activity was observed during ripening of apples. ${ }^{7}$ For aroma biosynthesis in mature apples (aldehyde and ester formation), MdLOX1 a was identified as the most promising candidate as recent investigations on LOX gene expression revealed an ample rise in MdLOX1a transcript abundance upon ethylene-induced fruit ripening., ${ }^{9,24}$ Ripeningdependent changes in the LOX pathway have already been discussed to account for the formation of aroma-relevant alcohol and ester volatiles. ${ }^{58}$

We propose that MdLOX1a is responsible for the majority of LOX activity found in intact apple fruits during the late stages of the ripening process based, among others, on the peculiar conformity of the regio- and stereochemistry of the hydroperoxide products formed by the recombinant and endogenous enzyme. Thus, LOX1:Md:1a is suggested to be involved in the generation of C6 aldehydes (primarily hexanal in the case of apple) and the formation of hexyl and possibly other straight-chain esters in intact, ripening fruit (Figure 7) whereas other LOXs primarily take part in the production of 'green note' aldehydes. In particular, the coincident ripening-dependent decline in the synthesis of unsaturated C6 aldehydes in disrupted tissue and associated esters ${ }^{7}$ and the decline in two of the putative 13-LOX genes, MdLOX6a and MdLOX6b, reported herein is also suggestive of the involvement of specific LOX genes in regulating the synthesis of disruptiondependent aldehydes.

In stored apple fruits, it seems that the degradation of LA by the $\beta$-oxidation pathway is impaired as high amounts of the intermediates escape as octane-1,3-diol ${ }^{75}$ (Figure 7). Likewise, apple fruit do not accumulate octanoic acid derived esters from LA but form abundant amounts of hexyl acetate and hexanoic acid derived esters. ${ }^{8}$ We assume that saturated and mono-unsaturated fatty acids are primarily degraded by $\beta$-oxidation in intact fruit, thus providing precursors for short, straight-chain esters, whereas the late steps of the $\beta$-oxidation process for polyunsaturated fatty acids such as LA and LnA are impaired (Figure 7). MdLOX1a activity might contribute to ester formation in intact fruit as it circumvents the metabolic block and channels short chain aldehydes into the biosynthetic pathway of aliphatic esters. 


\section{COMPETING INTERESTS}

The authors declare no conflict of interest.

\section{ACKNOWLEDGEMENTS}

The authors are grateful for the technical support provided by Fong-Chin Huang and Thomas Hoffmann. This research was financially supported by the Deutsche Forschungsgemeinschaft in frame of the project numbers AOBJ 575560 and AOBJ 575561.

\section{REFERENCES}

1 Song J, Forney CF. Flavor volatile production and regulation in fruit. Can J Plant Sci/ Rev Can Phytotech 2008; 88: 537-550.

2 Dixon J, Hewett EW. Factors affecting apple aroma/flavor volatile concentration a review. NZ J Crop Hort Sci 2000; 28: 155-173.

3 Paillard NMM. The flavor of apples pears and quinces. In: Morton ID, MacLeod AJ (eds.) Food Flavors Part C The Flavor of Fruits. Amsterdam: Elsevier, 1990: 1-42.

4 Mattheis JP, Fellman JK, Chen PM, Patterson ME. Changes in headspace volatiles during physiological development of Bisbee Delicious apple fruits. J Agric Food Chem 1991; 39: 1902-1906.

5 Altisent R, Echeverría G, Graell J, López L, Lara I. Lipoxygenase activity is involved in the regeneration of volatile ester-synthesizing capacity after ultra-low oxygen storage of 'Fuji' apple. J Agric Food Chem 2009; 57: 4305-4312.

6 Schwab W, Davidovich-Rikanati R, Lewinsohn E. Biosynthesis of plant-derived flavor compounds. Plant J 2008; 54: 712-732.

7 Contreras C, Beaudry R. Lipoxygenase-associated apple volatiles and their relationship with aroma perception during ripening. Postharv Biol Technol 2013; 82: $28-38$.

8 Rowan DD, Allen JM, Fielder S, Hunt MB. Biosynthesis of straight-chain ester volatiles in red delicious and granny smith apples using deuterium-labeled precursors. J Agric Food Chem 1999; 47: 2553-2562.

9 Schaffer RJ, Friel EN, Souleyre EJ et al. A genomics approach reveals that aroma production in apple is controlled by ethylene predominantly at the final step in each biosynthetic pathway. Plant Physiol 2007; 144: 1899-1912.

10 Sugimoto N, Jones D, Beaudry R. Changes in free amino acid content in 'Jonagold' apple fruit as related to branched-chain ester production ripening and senescence. J Amer Soc Hort Sci 2011; 136: 429-440.

11 Porta H, Rocha-Sosa M. Plant Lipoxygenases. Physiological and molecular features. Plant Physiol 2002; 130: 15-21.

12 Feussner I, Kuhn H, Wasternack C. Lipoxygenase-dependent degradation of storage lipids. Trends Plant Sci 2001; 6: 268-273.

13 Feussner I, Wasternack C. The lipoxygenase pathway. Annu Rev Plant Biol 2002; 53 : 275-297.

14 Casey R, West SI, Hardy D, Robinson DS, Wu Z, Hughes RK. New frontiers in food enzymology recombinant lipoxygenases. Trends Food Sci Technol 1999; 10: $297-$ 302.

15 Tressl R, Drawert F, Heimann W, Emberger R. Über die Biogenese von Aromastoffen bei Pflanzen und Früchten. VIII Mitteilung Zur Biogenese der in Bananen gefundenen Aromastoffe. Eur Food Res Technol 1970; 144: 4-12.

16 Chen G, Hackett R, Walker D, Taylor A, Lin Z, Grierson D. Identification of a specific isoform of tomato lipoxygenase (TomloxC) involved in the generation of fatty acidderived flavor compounds. Plant Physiol 2004; 136: 2641-2651.

17 Shin JH, Van K, Kim DH et al. The lipoxygenase gene family a genomic fossil of shared polyploidy between Glycine max and Medicago truncatula. BMC Plant Biol 2008; 8: 133.

18 Podolyan A, White J, Jordan B, Winefield C. Identification of the lipoxygenase gene family from Vitis vinifera and biochemical characterisation of two 13-lipoxygenases expressed in grape berries of Sauvignon Blanc. Funct Plant Biol 2010; 37: 767-784.

19 Riley J, Willemot C, Thompson JE. Lipoxygenase and hydroperoxide lyase activities in ripening tomato fruit. Postharvest Biol Technol 1996; 7: 97-107.

20 González-Agüero M, Troncoso S, Gudenschwager O, Campos-Vargas R, Moya-León MA, Defilippi BG. Differential expression levels of aroma-related genes during ripening of apricot (Prunus armeniaca L). Plant Physiol Biochem 2009; 47: 435-440.

21 Zhang B, Shen JY, Wei WW et al. Expression of genes associated with aroma formation derived from the fatty acid pathway during peach fruit ripening. $J$ Agric Food Chem 2010; 58: 6157-6165.

22 Han M, Zhang T, Zhao C, Zhi J. Regulation of the expression of lipoxygenase genes in Prunus persica fruit ripening. Acta Physiol Plant 2011; 33: 1345-1352.

23 Zhang B, Chen K, Bowen J et al. Differential expression within the LOX gene family in ripening kiwifruit. J Exp Bot 2006; 57: 3825-3836.

24 Vogt J, Schiller D, Ulrich D, Schwab W, Dunemann F. Identification of lipoxygenase (LOX) genes putatively involved in fruit flavor formation in apple (Malus $\times$ domestica). Tree Genet Genom 2013; 9: 1493-1511.
25 Park S, Sugimoto N, Larson MD, Beaudry R, van Nocker S. Identification of genes with potential roles in apple fruit development and biochemistry through large-scale statistical analysis of expressed sequence tags. Plant Physiol 2006; 141: 811-824.

26 Dunemann F, Ulrich D, Boudichevskaia A, Grafe C, Weber WE. QTL mapping of aroma compounds analyzed by headspace solid-phase microextraction gas chromatography in the apple progeny 'Discovery' $X^{\prime}$ Prima'. Mol Breeding 2009; 23: 501-521.

27 Liao Z, Chen M, Guo L et al. Rapid isolation of high-quality total RNA from Taxus and Ginkgo. Prep Biochem Biotechnol 2004; 34: 209-214.

28 Wang C, Wilkins T. A modified hot borate method significantly enhances the yield of high-quality RNA from cotton (Gossypium hirsutum L). Anal Biochem 1994; 223: 7-12.

29 Rozen S, Skaletsky H. Primer3 on the WWW for general users and for biologist programmers. In: Misener S, Krawetz S (eds.) Bioinformatics Methods and Protocols. Totowa, NJ: Humana Press, 1999: 365-386.

30 Yokoyama R, Nishitani K. A comprehensive expression analysis of all members of a gene family encoding cell-wall enzymes allowed us to predict cis-regulatory regions involved in cell-wall construction in specific organs of Arabidopsis. Plant Cell Physiol 2001; 42: 1025-1033.

31 Livak K, Schmittgen T. Analysis of relative gene expression data using real-time quantitative PCR and the $2^{-\Delta \Delta C T}$ method. Methods 2001; 25: 402-408.

32 Huang FC, Studart-Witkowski C, Schwab W. Overexpression of hydroperoxide lyase gene in Nicotiana benthamiana using a viral vector system. Plant Biotechnol J 2010; 8: 783-795.

33 Bradford MM. A rapid and sensitive method for the quantitation of microgram quantities of protein utilizing the principle of protein-dye binding. Anal Biochem 1976; 72: 248-254.

34 Arnold K, Bordoli L, Kopp J, Schwede T. The SWISS-MODEL workspace a web-based environment for protein structure homology modelling. Bioinformatics 2006; 22: 195-201.

35 Horton RM. PCR-mediated recombination and mutagenesis. Mol Biotechnol 1995; 3: 93-99.

36 Nakai K, Horton P. PSORT a program for detecting the sorting signals of proteins and predicting their subcellular localization. Trends Biochem Sci 1999; 24: 34-35.

37 Small I, Peeters N, Legeai F, Lurin C. Predotar A tool for rapidly screening proteomes for N-terminal targeting sequences. Proteomics 2004; 4: 1581-1590.

38 von Heijne G, Nishikawa K. Chloroplast transit peptides the perfect random coil? FEBS Lett 1991; 278: 1-3.

39 Earley K, Haag J, Pontes O et al. Gateway-compatible vectors for plant functional genomics and proteomics. Plant J 2006; 45: 616-629.

40 Brandizzi F, Snapp E, Roberts A, Lippincott-Schwartz J, Hawes C. Membrane protein transport between the endoplasmic reticulum and the Golgi in tobacco leaves is energy dependent but cytoskeleton independent evidence from selective photobleaching. Plant Cell 2002; 14: 1293-1309.

41 Sparkes I, Runions J, Kearns A, Hawes C. Rapid transient expression of fluorescent fusion proteins in tobacco plants and generation of stably transformed plants. Nature Protocols 2006; 1: 2019-2025.

42 Siedow JN. Plant lipoxygenase structure and function. Annu Rev Plant Biol 1991; 42: 145-188.

43 Mita G, Gallo A, Greco V et al. Molecular cloning and biochemical characterization of a lipoxygenase in almond (Prunus dulcis) seed. Eur J Biochem 2001; 268: 15001507.

44 Santino A, de Paolis A, Gallo A, Quarta A, Casey R, Mita G. Biochemical and molecular characterization of hazelnut (Corylus avellana) seed lipoxygenases. Eur J Biochem 2003; 270: 4365-4375.

45 Sloane DL, Leung R, Barnett J, Craik CS, Sigal E. Conversion of human 15lipoxygenase to an efficient 12-lipoxygenase the side-chain geometry of amino acids 417 and 418 determine positional specificity. Protein Eng 1995; 8: 275-282.

46 Hornung $E$, Walther $M$, Kühn H, Feussner I. Conversion of cucumber linoleate 13lipoxygenase to a 9-lipoxygenating species by site-directed mutagenesis. Proc Natl Acad Sci USA 1999; 96: 4192-4197.

47 Coffa G, Brash AR. A single active site residue directs oxygenation stereospecificity in lipoxygenases stereocontrol is linked to the position of oxygenation. Proc Natl Acad Sci USA 2004; 101: 15579-15584.

48 Coffa G, Imber AN, Maguire BC et al. On the relationships of substrate orientation hydrogen abstraction and product stereochemistry in single and double dioxygenations by soybean lipoxygenase- 1 and its Ala542Gly mutant. J Biol Chem 2005; 280: 38756-38766.

49 Boeglin WE, Itoh A, Zheng Y, Coffa G, Howe GA, Brash AR. Investigation of substrate binding and product stereochemistry issues in two linoleate 9-lipoxygenases. Lipids 2008; 43: 979-987.

50 Kuhn H, Saam J, Eibach S, Holzhütter HG, Ivanov I, Walther M. Structural biology of mammalian lipoxygenases enzymatic consequences of targeted alterations of the protein structure. Biochem Biophys Res Commun 2005; 338: 93-101. 
51 Farmaki T, Sanmartin M, Jimenez $P$ et al. Differential distribution of the lipoxygenase pathway enzymes within potato chloroplasts. J Exp Bot 2007; 58: 555-568.

52 Villatoro C, Echeverría G, Graell J, López ML, Lara I. Long-term storage of pink lady apples modifies volatile-involved eEnzyme activities consequences on production of volatile esters. J Agric Food Chem 2008; 56: 9166-9174.

53 Zhang B, Yin XR, Shen JY, Chen KS, Ferguson IB. Volatiles production and lipoxygenase gene expression in kiwifruit peel and flesh during fruit ripening. J Am Soc Hort Sci 2009; 134: 472-477.

54 Dunemann F, Ulrich D, Malysheva-Otto $L$ et al. Functional allelic diversity of the apple alcohol acyl-transferase gene MdAAT1 associated with fruit ester volatile contents in apple cultivars. Mol Breeding 2012; 29: 609-625.

55 Echeverría G, Correa E, Ruiz-Altisent M, Graell J, Puy J, López L. Characterization of Fuji apples from different harvest dates and storage conditions from measurements of volatiles by gas chromatography and electronic nose. J Agric Food Chem 2004; 52: 3069-3076.

56 Altisent R, Graell J, Lara I, López L, Echeverría G. Increased straight-chain esters content after ultra low oxygen storage and its relation to the lipoxygenase system in 'Golden Reinders ${ }^{\circledR \prime}$ apples. Eur Food Res Technol 2011; 232: 51-61.

57 Li DP, Xu YF, Sun LP et al. Salicylic acid ethephon and methyl jasmonate enhance ester regeneration in 1-MCP-treated apple fruit after long-term cold storage. J Agric Food Chem 2006; 54: 3887-3895.

58 Defilippi BG, Dandekar AM, Kader AA. Relationship of ethylene biosynthesis to volatile production related enzymes and precursor availability in apple peel and flesh tissues. J Agric Food Chem 2005; 53: 3133-3141.

59 Ferenczi A, Song J, Tian M, Vlachonasios K, Dilley D, Beaudry R. Volatile ester suppression and recovery following 1-methylcyclopropene application to apple fruit. J Am Soc Hort Sci 2006; 131: 691-701.

60 Davenport JB. Studies in the natural coating of apples. V Unsaturated and minor saturated acids of the cuticle oil. Aust J Chem 1960; 13: 411-415.

61 Block MA, Dorne AJ, Joyard J, Douce R. Preparation and characterization of membrane fractions enriched in outer and inner envelope membranes from spinach chloroplasts. J Biol Chem 1983; 258: 13282-13286.

62 Somerville C, Browse J, Jaworski J, Ohlrogge J. Lipids. In: Buchanan B, Gruissem W, Jones R (eds.) Biochemistry and Molecular Biology of Plants. 1st ed. Rockville, MD: American Society of Plant Biologists, 2000: 456-527.

63 Moyer JC, Aitken HC. Apple juice. In: Nelson E, Tressler DK (eds.) Fruit and Vegetable Juice Processing Technology. 3rd ed. Westport, CT: AVI Publishing, 1980: 212-267.

64 Feys M, de Mot R, Naesens W, Tobback P. Solubilization partial purification and properties of lipoxygenase from apples. Eur Food Res Technol 1982; 174, 360-365.
65 Kim IS, Grosch W. Partial purification of a lipoxygenase from apples. J Agric Food Chem 1979; 27: 243-246.

66 Grosch W, Laskawy G, Fischer KH. Positions-Spezifität der Peroxidierung von Linolund Linolensäure durch Homogenate aus Äpfeln und Birnen. Eur Food Res Technol 1977; 163: 203-205.

67 Beuerle T, Schwab W. Metabolic profile of linoleic acid in stored apples formation of 13(R)-hydroxy-9(Z)11(E)-octadecadienoic acid. Lipids 1999; 34: 375-380.

68 van Os CPA, Rijke-Schilder GPM, Vliegenthart JFG. 9- $\mathrm{L}_{R}$-linoleyl hydroperoxide a novel product from the oxygenation of linoleic acid by type-2 lipoxygenases from soybeans and peas. Biochim Biophys Acta 1979; 575: 479-484.

69 Palmieri-Thiers C, Canaan S, Brunini V et al. A lipoxygenase with dual positional specificity is expressed in olives (Olea europaea $\mathrm{L}$ ) during ripening. Biochim Biophys Acta 2009; 1791: 339-346.

70 Gardner HW, Grechkin AN. Biocatalysis by the plant lipoxygenase pathway: oxygenated fatty acid production and hydroperoxide lyases. In: Kuo TM, Gardner HW (eds.) Lipid Biotechnology. New York: Marcel Dekker Inc., 2002: 157-182.

71 Kajiwara T, Sekiya J, Asano M, Hatanaka A. Enantioselectivity of enzymatic cleavage reaction of 13-hydroperoxylinolenic acid to $\mathrm{C}_{6}$-aldehyde and $\mathrm{C}_{12}$-oxo acid in tea chloroplasts. Agric Biol Chem 1982; 46: 3087-3088.

72 Gardner HW, Weisleder D, Plattner RD. Hydroperoxide lyase and other hydroperoxide-metabolizing activity in tissues of soybean Glycine max. Plant Physiol 1991; 97: 1059-1072.

73 Drawert F, Heimann W, Emberger R, TressI R. Über die Biogenese von Aromastoffen bei Pflanzen und Früchten. II Enzymatische Bildung von Hexen-(2)-al-(1) Hexanal und deren Vorstufen. Justus Liebigs Ann Chem 1966; 694: 200-208.

74 Schreier P, Lorenz G. Separation partial purification and characterization of a fatty acid hydroperoxide cleaving enzyme from apple and tomato fruits. $Z$ Naturforsch Sect C 1982; 37: 165-173.

75 Beuerle T, Schwab W. Biosynthesis of octane-13-diol in apple fruit. Phytochemistry 1997; 45: 1153-1155.

(c) (i) (2) This work is licensed under a Creative Commons Attribution-

BY NG SA NonCommercial-ShareAlike 3.0 Unported License. The images or other third party material in this article are included in the article's Creative Commons license unless indicated otherwise in the credit line; if the material is not included under the Creative Commons license, users will need to obtain permission from the license holder to reproduce the material. To view a copy of this license, visit http://creativecommons.org/ licenses/by-nc-sa/3.0/

Supplemental Information for this article can be found on the Horticulture Research website (http://www.nature.com/hortres). 